ARTICLE

https://doi.org/10.1038/s41467-019-11409-0

\title{
Class Ila HDACs regulate learning and memory through dynamic experience-dependent repression of transcription
}

Yongchuan Zhu',2, Min Huang 1,2,3, Eric Bushong (10 4, Sebastien Phan4, Marco Uytiepo 1,2, Elizabeth Beutter 1,2, Daniel Boemer ${ }^{1,2}$, Kristin Tsui ${ }^{1,2}$, Mark Ellisman ${ }^{4}$ \& Anton Maximov ${ }^{1,2}$

The formation of new memories requires transcription. However, the mechanisms that limit signaling of relevant gene programs in space and time for precision of information coding remain poorly understood. We found that, during learning, the cellular patterns of expression of early response genes (ERGs) are regulated by class Ila HDACs 4 and 5, transcriptional repressors that transiently enter neuronal nuclei from cytoplasm after sensory input. Mice lacking these repressors in the forebrain have abnormally broad experience-dependent expression of ERGs, altered synaptic architecture and function, elevated anxiety, and severely impaired memory. By acutely manipulating the nuclear activity of class Ila HDACs in behaving animals using a chemical-genetic technique, we further demonstrate that rapid induction of transcriptional programs is critical for memory acquisition but these programs may become dispensable when a stable memory is formed. These results provide new insights into the molecular basis of memory storage.

\footnotetext{
${ }^{1}$ Department of Neuroscience, The Scripps Research Institute, La Jolla, CA 92037, USA. ${ }^{2}$ The Dorris Neuroscience Center and The Scripps Research Institute, La Jolla, CA 92037, USA. ${ }^{3}$ The Kellogg School of Science and Technology, The Scripps Research Institute, La Jolla, CA 92037, USA. ${ }^{4}$ National Center for Microscopy and Imaging Research, University of California, San Diego, CA, USA. Correspondence and requests for materials should be addressed to A.M. (email: amaximov@scripps.edu)
} 
S ensory exprience activates transcriptional programs in the brain that affect neuronal wiring, synaptic function, and memory formation ${ }^{1,2}$. The first waves of dynamic experience-dependent changes in neuronal transcriptomes arise from transient expression of early response genes (ERGs), which are only induced in subsets of cells in a given environment ${ }^{3-5}$. Most ERGs encode transcription factors (TFs) that exert their physiological effects through downstream target genes ${ }^{1}$. Labeling for native ERG products and expression of exogenous molecules under the control of ERG promoters is now broadly used in neuroscience to identify and manipulate neuronal ensembles recruited for particular cognitive tasks ${ }^{6-8}$. For example, memory traces can be artificially retrieved via optogenetic stimulation of ERG-positive cells that were permanently tagged in cortical and hippocampal circuits during learning ${ }^{9-11}$.

Although cellular patterns of ERG induction are thought to reflect sparse coding of information in the brain ${ }^{3,11,12}$, it remains unclear how these patterns are controlled in time and space. Gene expression is orchestrated by TFs and transcriptional repressors that either silence discrete loci or globally alter the state of nuclear chromatin $^{13}$. Several TFs that mediate activity-dependent synthesis of neuronal mRNAs have been characterized ${ }^{1,14,15}$, but the extent and molecular mechanisms of negative regulation of this process are poorly understood. It is also unclear how ERGs contribute to different aspects of information processing, including memory acquisition, consolidation, storage, and retrieval, as the current knowledge about ERG function in mammals largely stems from experimental paradigms that suffer from poor temporal control ${ }^{16-19}$.

Class IIa histone deacetylases (HDACs) are a small family of chromatin-binding proteins that shuttle between the nucleus and cytoplasm in a calcium- and phosphorylation-dependent manner ${ }^{20-23}$. The vertebrate class IIa HDACs interact with class I HDACs, SMRT/NCoR co-repressor complexes, and various TFs via their $\mathrm{N}$ - and $\mathrm{C}$-terminal domains, and exhibit intrinsically low enzymatic activity on $\varepsilon-N$-acetyl lysine substrates due to evolutionary inactivation of the catalytic site ${ }^{24}$. Genetic studies have suggested that the class IIa HDAC isoform, HDAC4, is necessary for normal cognition in Drosophila, mice, and humans ${ }^{25-28}$. Yet, prior attempts to define the functional role of HDAC4 in the central nervous system have led to conflicting findings. On the one hand, HDAC4 is capable of binding to TFs essential for synaptic plasticity and memory, CREB, MEF2, and SRF, and its constitutively nuclear gain-of-function mutants repress numerous neuronal genes in vitro ${ }^{23,25,29}$. Puzzlingly, the majority of central neurons retain HDAC4 in the cytoplasm ${ }^{23,25,30}$ and Hdac4deficient mice have been reported to have no detectable abnormalities in mRNA levels in the brain ${ }^{26,31}$. Despite recent reports that HDAC4 irreversibly enters neuronal nuclei under the pathological conditions, such as Ataxia telangiectasia and Parkinson's disease $\mathrm{s}^{32,33}$, the physiological significance of the nuclear import of this protein in normal circuits is unclear.

Herein, we elucidated the function of class IIa HDACs by combining mouse genetics with imaging, genomic, electrophysiological, and behavioral readouts. We found that HDAC4 and its close homolog, HDAC5, redundantly act in the same pathway that is essential for spatiotemporal control of ERG programs during memory encoding. We then developed and applied a new chemical-genetic technique that leverages these findings and permits acute transcriptional repression in brains of behaving animals with a small molecule that rapidly stabilizes HDAC4 in neuronal nuclei. Our results demonstrate that induction of ERGs is necessary for learning, but these genes may not be required after a memory is formed. This work provides insight into the molecular basis of memory storage.

\section{Results}

To explore the possibility that HDAC4 regulates network plasticity during learning, we examined the subcellular localization of this protein in wild-type (WT) mice after subjecting them to contextual fear conditioning (CFC), a neurobehavioral paradigm that prompts the acquisition of associative fear memory (Fig. 1a, b) ${ }^{3,9}$. This was accomplished by immunolabeling of brain sections with an antibody whose specificity was validated in Hdac4 knockouts. Unlike control home-caged mice, trained animals (1.5 h post CFC) had a pronounced nuclear accumulation of HDAC4 in $26 \%$ to $58 \%$ of cells in the somatosensory cortex, areas CA1/CA3 of the hippocampus, and the dentate gyrus, brain regions known to be critical for memory encoding $2,11,34$. This effect was reversed by $6 \mathrm{~h}$ after CFC, indicating that experiencedependent nuclear import of HDAC4 is transient (Fig. 1c, $\mathrm{d}$ and Supplementary Fig. 1).

We then performed double-labeling with antibodies against HDAC4 and Fos to assess the localization of HDAC4 in sparsely distributed Fos-positive neurons that are thought to represent cellular substrates of memory engrams ${ }^{3,11}$. Remarkably, $\sim 80 \%$ of neurons with detectable Fos immunoreactivity in the hippocampi of fear-conditioned mice ( $1.5 \mathrm{~h}$ post CFC) retained HDAC4 in the cytoplasm, raising the possibility that HDAC4 restricts the expression of Fos and possibly other ERGs in circuits essential for memory storage (Fig. 1e, f).

We and others have previously reported that HDAC4 binds to neuronal chromatin and that prolonged overexpression of a constitutively nuclear phosphorylation-deficient HDAC4 mutant (HDAC4-3SA, Supplementary Fig. 2a) downregulates multiple neuronal genes in vitro $23,25,29$. However, the capacity of HDAC4 for repressing activity-dependent transcription has not been established. We therefore infected cultured cortical neurons with lentiviruses (LVs) encoding WT HDAC4 or HDAC4-3SA and measured the levels of RNAs that were isolated prior to or after brief depolarization with $\mathrm{KCl}$, which stimulates ERGs. Genomewide mRNA profiling (RNA sequencing (RNA-seq)) and quantitative real-time PCR ( $\mathrm{qPCR}$ ) analyses of individual transcripts consistently demonstrated that HDAC4-3SA interfered with induction of Fos and other classical ERGs Egr2, Egr3, Egr4, Nr4a1, Nr4a3, Arc, and Npas41,4 (Fig. 1g, h, Supplementary Figs. 2 and 3, and Supplementary Data File 1).

In addition to HDAC4, neurons in the adult brain abundantly express another class IIa HDAC isoform, HDAC5 ${ }^{25}$. This isoform has been recently shown to influence reward seeking by interacting with Npas4 in the nucleus accumbens ${ }^{35}$, but its functional role in other circuits is also unclear. Although we were unable to monitor the dynamic redistribution of native HDAC5 due to insufficient quality of available antibodies, deep sequencing revealed that virtually all ERGs were repressed in cortical cultures by constitutively nuclear HDAC5-3SA mutant as well (Fig. 1g, right panel). Furthermore, the total pools of genes affected by each of the two class IIa HDAC isoforms largely overlapped, raising the possibility that these isoforms are functionally redundant (Supplementary Fig. 2).

To gain a mechanistic insight into how HDACs 4 and 5 silence ERGs, we monitored the expression of enhanced green fluorescent protein (EGFP) under the control of the Arc-based promoter, E-SARE. This short synthetic promoter contains DNAbinding sites for activity-dependent TFs essential for ERG response in the brain, CREB, MEF2, and SRF, and exhibits tight dependence on neuronal excitation in vitro and in vivo ${ }^{36}$. Again, $\mathrm{KCl}$-induced expression of E-SARE:EGFP was potently blocked in dissociated cultures by the nuclear 3SA mutants, whereas WT cytoplasmic constructs had no effect (Fig. 1i, j and Supplementary Fig. 4). These results imply that class IIa HDACs abolish the ERG transcription through inhibition of TFs on gene promoters. 
a
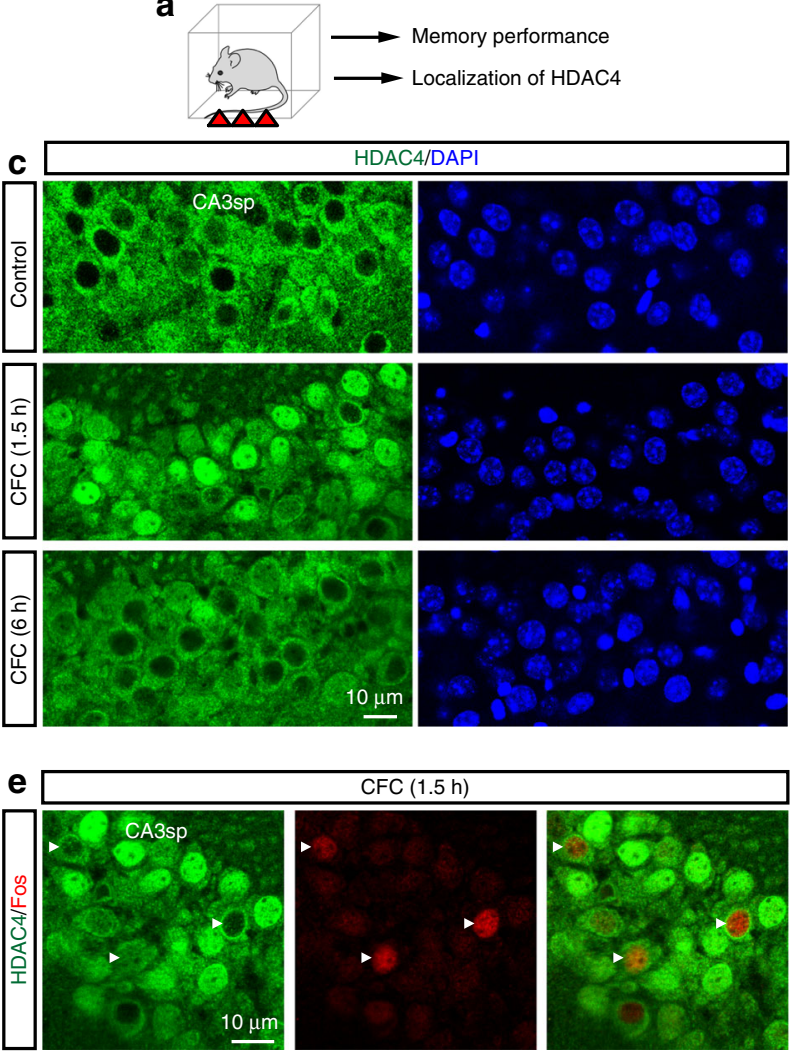

g
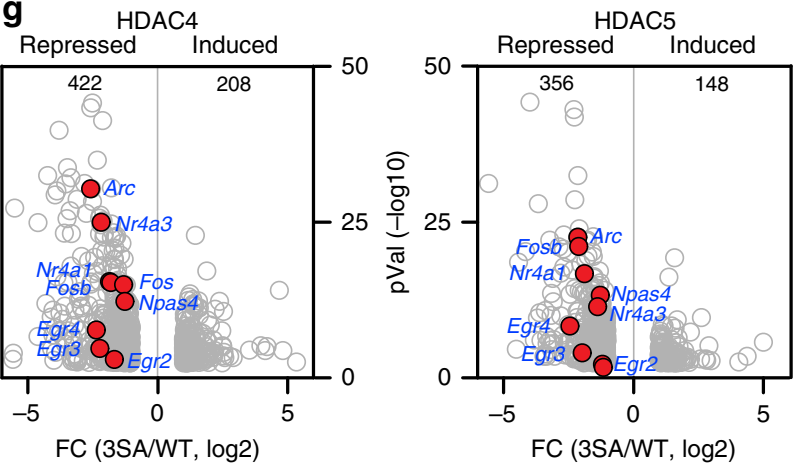

i
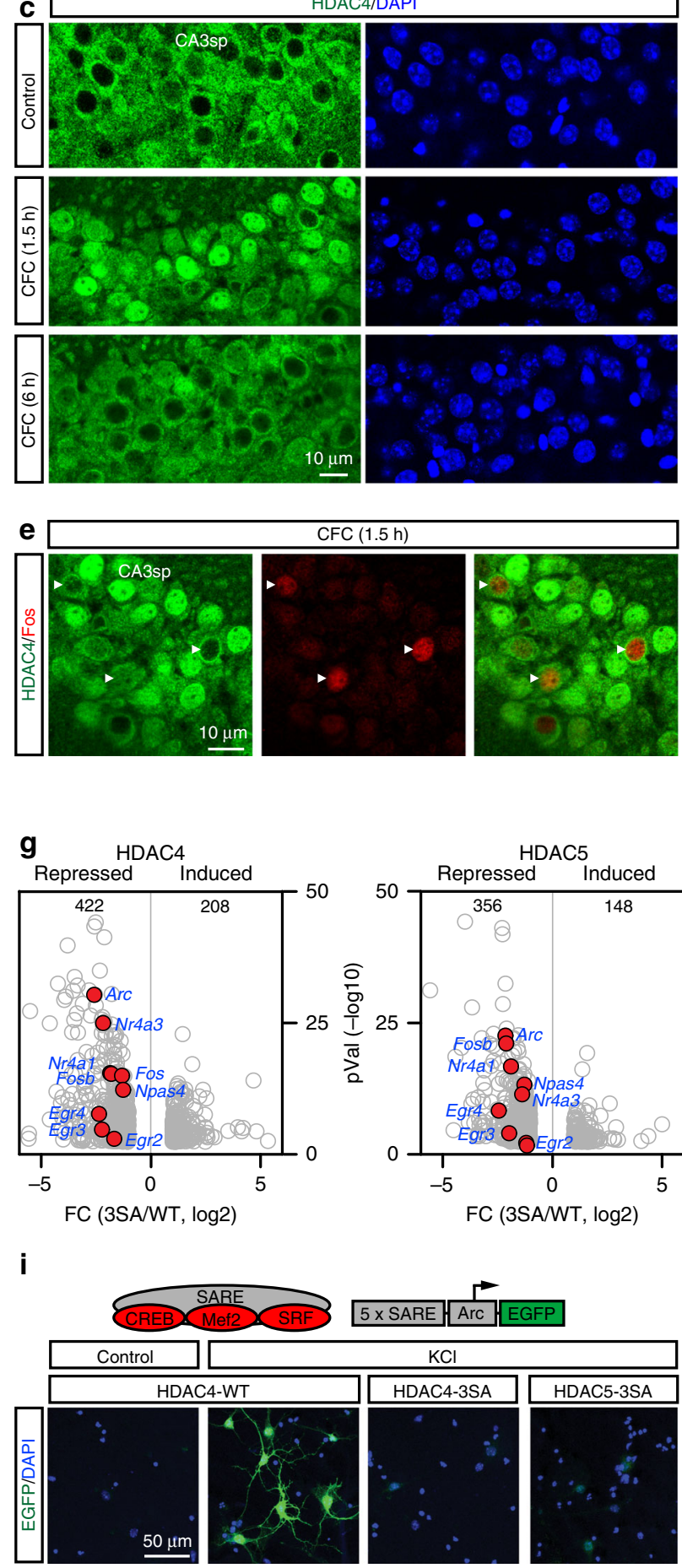

f

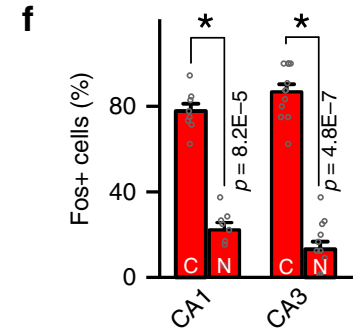

h

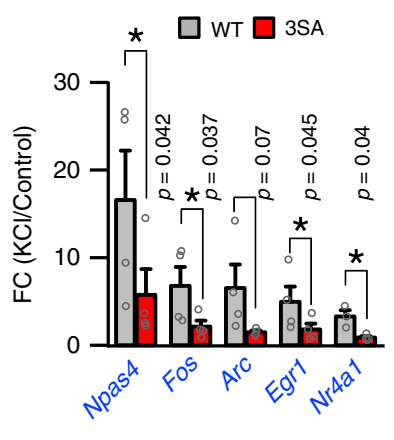

j

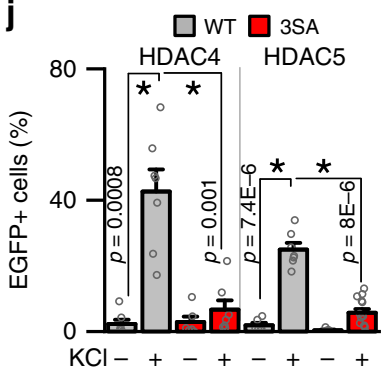

b
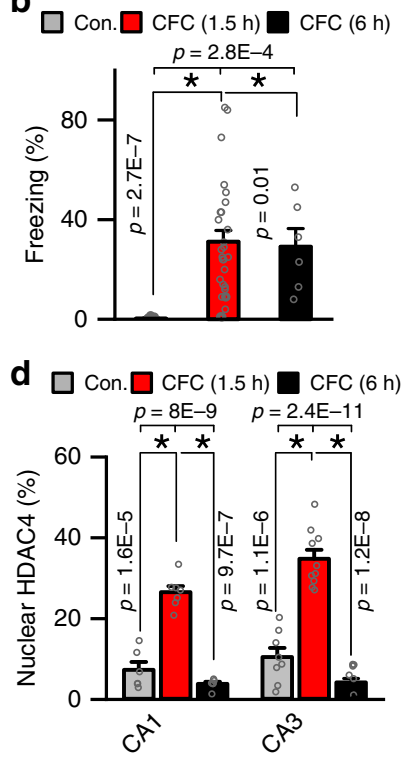

To determine whether HDACs 4 and 5 act in the same pathway in intact circuits, we generated double knockout (DKO) mice that lacked both repressors in glutamatergic neurons throughout the forebrain. These animals carried the previously described loxP-flanked ("floxed") Hdac4 alleles, constitutive Hdac5 knockout alleles, and a well-characterized Cre knock-in that drives loxP recombination at mid-gestation in progenitors of the Emx1 lineage that give rise to excitatory neurons and astrocytes $^{37-40}$. (Hdac4flox/flox/Hdac5-/-/EmxI IRES-Cre) (Supplementary Fig. 5a-c). We reasoned that recombination of Hdac4 in glia should not be problematic, as this gene is predominantly expressed in the neurons ${ }^{25,41}$. The homozygote DKOs were born at the expected Mendelian ratio and had regular lifespans. However, they could be easily distinguished from WT littermates based on fully penetrant hindlimb clasping in the tail suspension test. In contrast, the clasping was undetectable in mice 
Fig. 1 HDAC4 transiently enters neuronal nuclei during associative learning and represses ERGs. a-f Subcellular distribution of native HDAC4 in the hippocampus of wild-type p60 mice before and after contextual fear conditioning (CFC). a Experimental design. b Short-term memory performance was assessed by measuring freezing in the same context. Control, $n=11$ mice; CFC (1.5 h), $n=27$; CFC ( 6 h), $n=6$. c Images of HDAC4 immunofluorescence in area CA3sp of DAPI-stained brain sections. See also Supplementary Fig. 1. d Averaged percentages of cells with nuclear HDAC4 in CA1sp and CA3sp. $n=4$ mice/group (plotted data points are from individual sections). e Brain sections from fear-conditioned animals ( $1.5 \mathrm{~h}$ post CFC) were co-stained for HDAC4 and Fos. f Averaged fractions of Fos-positive cells with cytoplasmic (C) and nuclear (N) HDAC4 in indicated hippocampal subfields (1.5 h post (FC). $n=3$ mice. $\mathbf{g}$-j Effects of recombinant class Ila HDACs on activity-dependent transcription in primary cortical cultures. Neurons were infected at 2 days in vitro (DIV2) with lentiviruses (LVs) that express wild-type HDAC4/5 cDNAs or constitutively nuclear 3SA mutants. $\mathbf{g}$ Transcripts were isolated at DIV7 after 30 min depolarization with $\mathrm{KCl}(50 \mathrm{mM})$ and surveyed by deep sequencing. Volcano plots of differentially expressed genes from two independent RNA-seq experiments are shown. Fold changes are represented as log2 of 3SA/WT ratio for each HDAC isoform. ERGs are marked in red. See also Supplementary Figs. 2, 3 and Data File 1. h Depolarization-induced expression of ERGs was assessed by quantitative real-time PCR. Averaged FC values from four independent experiments are plotted as $\mathrm{KCl}$ /Control ratio. $\mathbf{i}$, $\mathbf{j}$ Indicated class Ila HDAC LVs were co-infected with the reporter of activitydependent transcription, E-SARE:EGFP. i Schematic diagram of E-SARE:EGFP and confocal images of EGFP fluorescence in control and KCl-depolarized neurons at DIV7. See also Supplementary Fig. 4. j Summary graphs of reporter induction. $n=3$ cultures/condition. All quantifications are represented as Mean \pm SEM (error bars). ${ }^{*} p<0.05$ (defined by ANOVA and/or $t$-test). Actual $p$-values are indicated in each graph (vertical for $t$-test and horizontal for ANOVA)

deficient for individual isoforms (Hdac4flox/flox $/ E m x 1^{\text {IRES-Cre }}$ and $H d a c 5^{-/}$), indicating that HDACs 4 and 5 can compensate for each other in vivo (Fig. 2a, b).

To address the concern that leg clasping of DKO mutants is due to major developmental defects or neurodegeneration, we carried out immunofluorescent imaging of brain sections that were labeled with antibodies for ubiquitous and excitatory neuron subtype-specific markers, NeuN, Cux1, Ctip2, Prox1, and Calbindin. The brains of adult DKOs had undistorted anatomies with appropriate lamination of cortical columns and the hippocampus, and no apparent increase in apoptosis (Fig. 2c-e and Supplementary Fig. 5d). We also examined the cellular architecture of the adult hippocampus by using single-cell sequencing (scRNA-seq) on the 10x Genomic platform. Unbiased analysis of 11,180 and 12,338 cells from dissociated hippocampi of three pairs of control and DKO mice, respectively, showed no changes in the abundance and clustering of neurons, astrocytes, oligodendrocytes, microglia, oligodendrocyte progenitor cells (OPCs), endothelial cells, and mural cells, further suggesting that cellular differentiation and survival were unaffected (Supplementary Data File 2).

Next, we investigated the consequences of HDAC4 and HDAC5 loss on gene expression levels by deep sequencing of libraries prepared from pooled hippocampal mRNAs. We chose this approach instead of singe-cell sequencing, as our scRNA-seq datasets had limited coverage at the expense of large cell numbers. Quantitative analysis of conventional RNA-seq reads with a statistical cutoff at $p<0.05$ identified 1451 differentially expressed genes, albeit only $\sim 14 \%$ of transcripts were up- or downregulated in class IIa HDAC-deficient animals by more than 2-fold (133 and 75, respectively). As expected, DKOs had a strong depletion of mRNAs of Hdac4 and Hdac5 (Fig. $2 \mathrm{f}$ and Supplementary Data File 3). Although the entire pool of affected genes was diverse, the STRING pathway analysis highlighted several prominent clusters of molecules required for synaptic function, network plasticity, and memory formation, including various receptors, ion channels, and scaffolds, proteins that mediate membrane trafficking, and, curiously, multiple adenylate cyclases and phosphodiesterases involved in cAMP signaling (Fig. $2 \mathrm{~g}$ and Supplementary Fig. 6).

As the majority of ERGs encode $\mathrm{TFs}^{1}$, these ERGs are repressed by HDACs 4 and 5 in vitro (Fig. 1), and each event of transient nuclear import of repressors likely has negligible effect on global expression levels of genes with long-lasting mRNAs, the observed molecular changes in brains of DKO mice may reflect a history of aberrant experience-dependent transcription. To test how loss of HDACs 4 and 5 affects the induction of
ERGs, we subjected DKOs to CFC and performed immunolabeling for Fos and Egr1. We imaged area CA3 of the hippocampus where background expression of these genes in homecaged animals is particularly low ${ }^{16}$. Consistent with observed CFC-induced redistribution of HDAC4 to neuronal nuclei in WT animals and analysis of repressor activity in neuronal cultures, DKOs had a $\sim 2$-fold increase in numbers of Fos- and Egr1-positive cells, although their contextual freezing responses were comparable to normal littermates (Fig. $2 \mathrm{~h}-\mathrm{j}$ ). Likewise, qPCR measurements of transcripts isolated from the whole hippocampi of fear-conditioned mice showed that DKOs had increased mRNA levels of Fos, Egr1, Nr4a1, Arc, and Npas4 shortly after CFC, and this increase persisted for several hours (Fig. 2k). These results demonstrate that class IIa HDACs are necessary for spatiotemporal control of ERG programs during memory encoding.

We systematically analyzed the behavior of DKO mutants at p60 to evaluate their motor skills, sensory processing, and ability to perform various cognitive tasks. Mice deficient for individual repressor isoforms (Hdac4flox/flox/EmxIIRES-Cre and $H \mathrm{dac5}^{-/-}$) were examined in parallel to further define the extent, or lack thereof, of functional redundancy. Despite clasping legs when lifted by tails, DKOs were hardly distinguishable from littermate controls in the standard laboratory environment. However, quantitative measurements of performance in several setups demonstrated that these animals were anxious and they had severely diminished capacity for acquiring new memories. The anxiety-like phenotype was apparent in dark/light transfer tests and analysis of activity in square open fields where DKOs preferred to remain in the dark and avoided the centers of open areas (Fig. 3a-c). These abnormalities were not due to aberrant vision or locomotion, as demonstrated by measurements of head tracks in the optomotor test, total travel distances, and overall horizontal activity (Fig. $3 \mathrm{~d}$ and Supplementary Fig. 7). When their spatial memory was monitored in the circular Barnes maze ${ }^{42}$, DKOs were able to identify the escape path during training sessions but showed no target discrimination in the subsequent probe test of memory recall (Fig. $3 \mathrm{e}-\mathrm{g}$ ). In addition, DKOs had virtually no fear extinction, as assessed for a week after CFC on a daily basis by quantifying freezing in the same context (Fig. 3h). The latter also indicates impaired learning rather than inability to erase an existing fear memory ${ }^{43}$. Notably, none of these phenotypes could be detected in single HDAC4 and HDAC5 KOs (Fig. 3a-c, g, h). It is also noteworthy that the outcomes of spatial and fear memory tests were not merely associated with anxiety and/or aberrant locomotion of DKO mice, as evidenced by quantifications of 

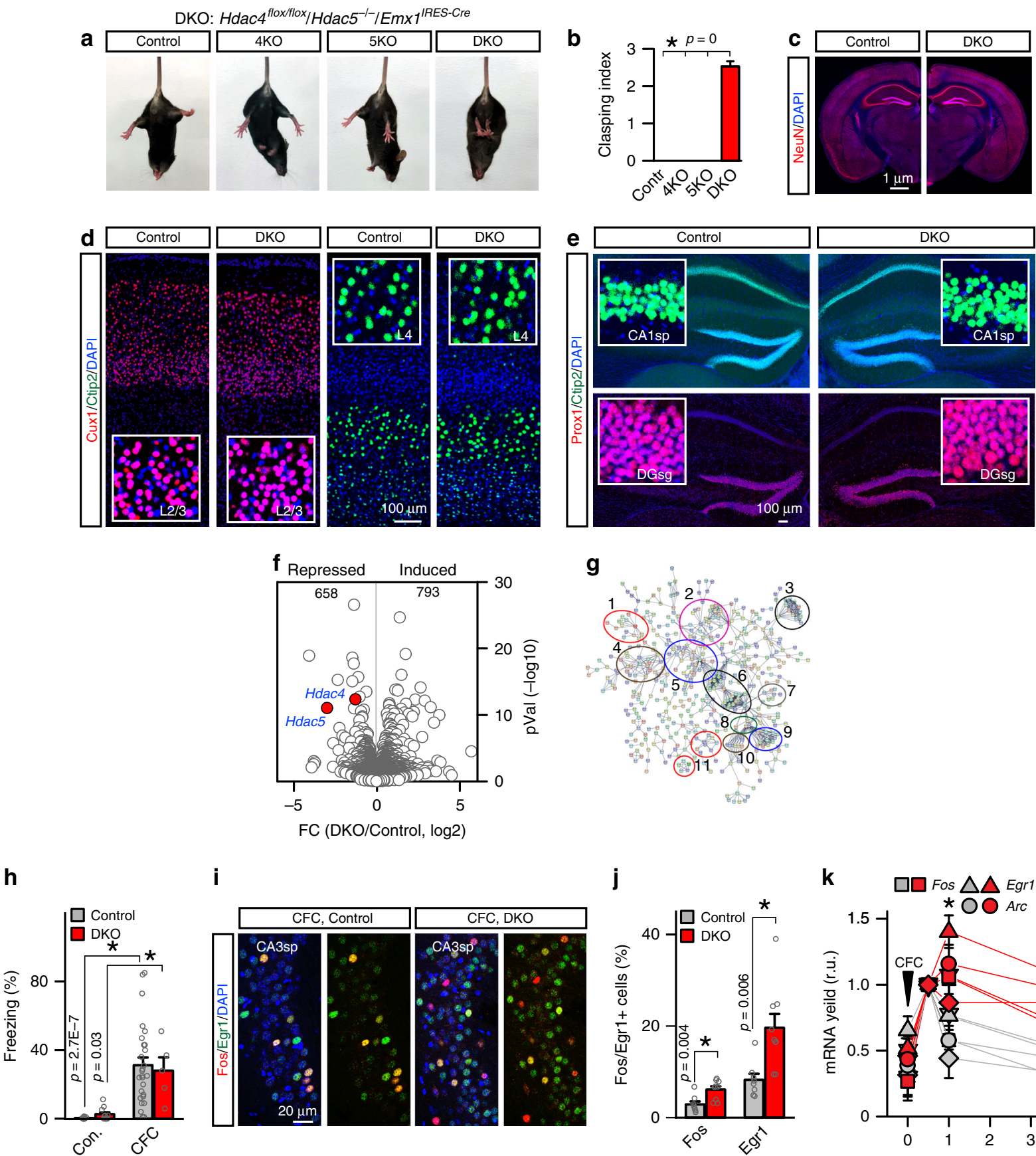

i

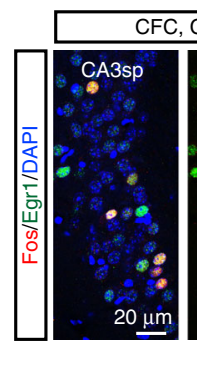

j

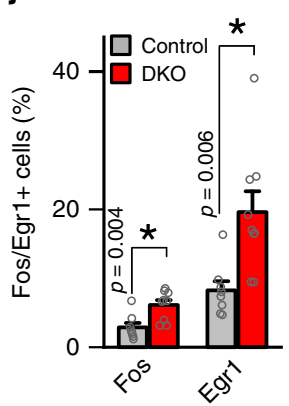

k

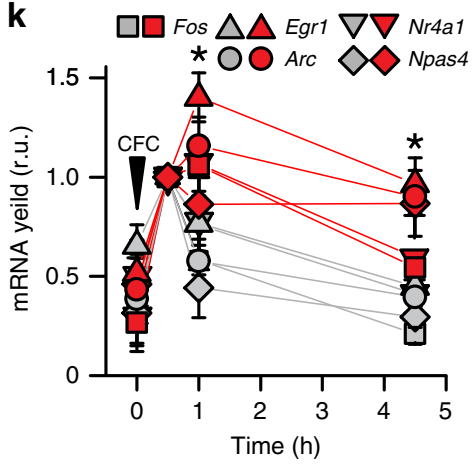

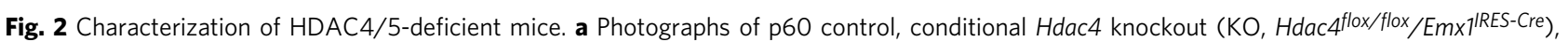
constitutive Hdac5 KO (Hdac5 $/-)$, and Hdac4/5 double knockout (DKO, Hdac4flox/flox $/$ Hdac5 $/-/$ Emx $7^{\text {RESS-Cre }}$ ) mice subjected to tail suspension test. b Averaged leg clasping indexes. $n=20$ mice/genotype. c Gross brain anatomies of $p 60$ control and DKO mice. Coronal brain sections were stained with DAPI and the antibody to a pan-neuronal marker, NeuN. See also Supplementary Fig. 5. d, e, Sections were labeled with antibodies against layer/subtypespecific markers of excitatory neurons, Cux1, Ctip2, and Prox1. Images of the primary somatosensory cortex (d) and the hippocampus (e) are shown. Higher-magnification frames are displayed in inserts. $\mathbf{f}, \mathbf{g}$ RNA-seq analysis of gene expression in the hippocampus at p60. Volcano plot (f) and the STRING network of differentially expressed transcripts $(\mathbf{g})$ are shown ( $n=3$ mice/genotype). The following clusters are marked: (1) neurotransmitter receptors and calcium channels; (2) cytoskeleton and scaffolds; (3) protein turnover; (4) membrane trafficking; (5) calcium signaling; (6) G proteins and G proteincoupled receptors; (7) MAP kinases; (8) adenylate cyclases; (9) dopamine and hormone receptors; (10) phosphodiesterases. See also Supplementary Fig. 6 and Data Files 2 and 3. h-k Expression of ERGs in the hippocampus during associative learning. $\mathbf{h}$ Analysis of short-term memory performance, assessed by measuring freezing in the same context $1.5 \mathrm{~h}$ post CFC. Control/Control, $n=11$; Control/CFC, $n=27$; DKO/Control, $n=11 ; \mathrm{DKO} / \mathrm{CFC}, n=5$. i Images of CA3sp of fear-conditioned control and DKO mice (1.5 h post CFC). Sections were labeled with DAPI and antibodies against Fos and Egr1. j Quantifications of Fos/Egr1-positive cells in CA3sp. $n=3$ mice/genotype. $\mathbf{k}$ Transcripts were isolated from hippocampi at indicated time points after CFC and expression levels of various ERGs were measured by QPCR. Values from four to five mice per genotype/time point are normalized to 30 min post CFC to highlight the prolonged window of ERG expression in DKOs. ${ }^{*} p<0.05$ ( $t$-test). The color coding is the same as in $\mathbf{j}$. All quantifications are represented as Mean \pm SEM (error bars). ${ }^{\star} p<0.05$ (defined by ANOVA and/or $t$-test) 
a
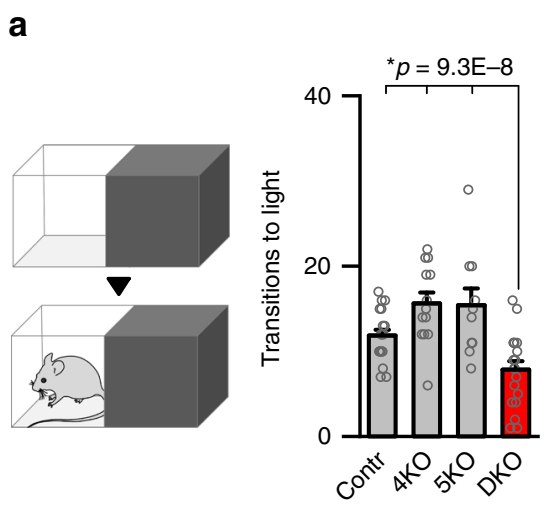

b

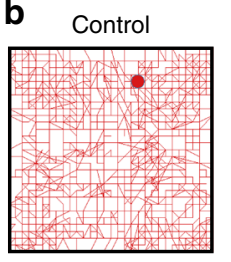

$5 \mathrm{KO}$

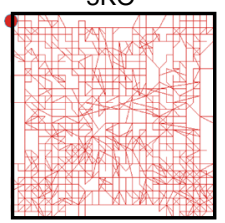

$4 \mathrm{KO}$

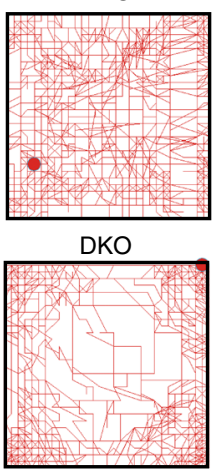

c

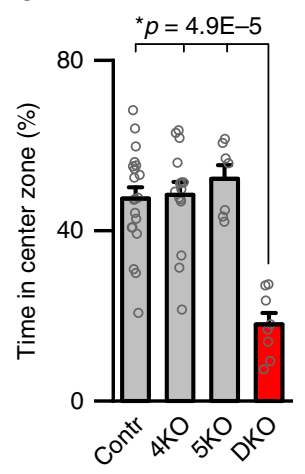

d

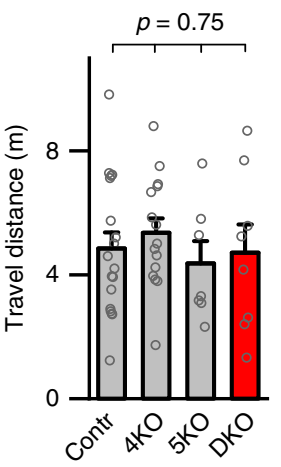

e

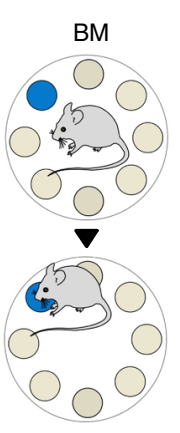

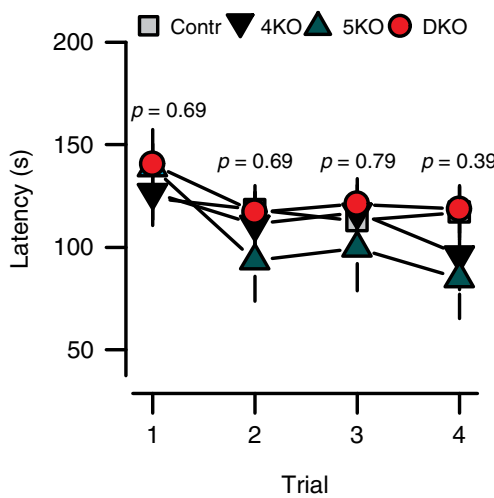

Trial f

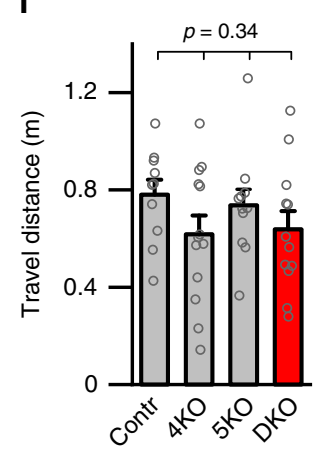

i

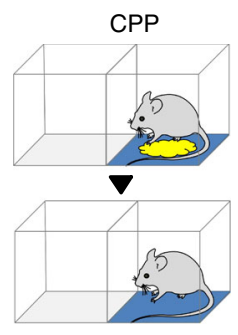

g

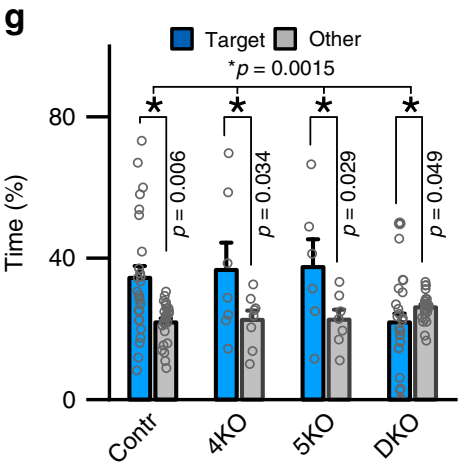

h

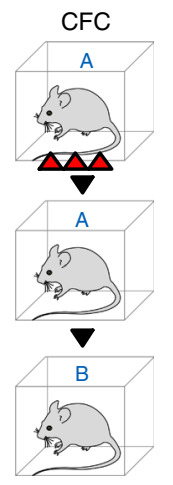

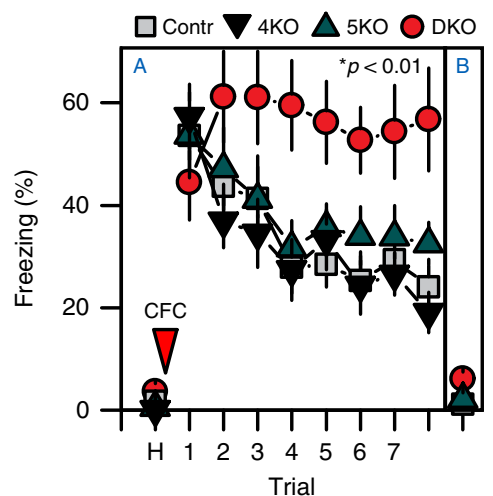

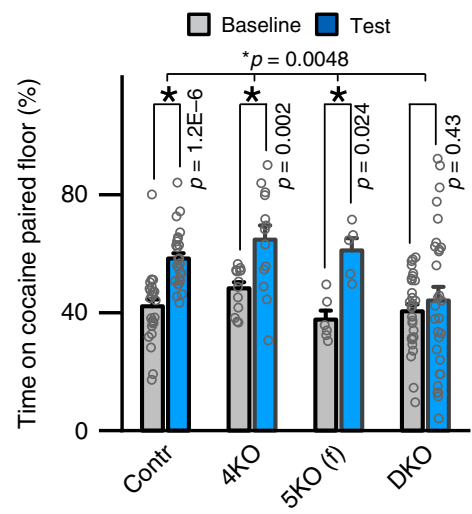

Fig. 3 Behavior of class Ila HDAC-deficient mice. Animals of indicated genotypes were examined at p60. See also Supplementary Fig. 7 and Methods. a Analysis of anxiety-like behavior in the dark-light transfer test. Schematics of experimental setup and averaged transitions to light are shown. Control: $n=19$ mice; 4KO: $n=13 ; 5 K O: n=10 ;$ DKO: $n=19$. b-d Locomotor activity in the open field setup. $\mathbf{b}$ Typical tracks during 30 min sessions. $\mathbf{c}$ Averaged times spent in the center zone. $\mathbf{d}$ Total travel distances. Control: $n=20 ; 4 \mathrm{KO}: n=15 ; 5 \mathrm{KO}: n=7 ;$ DKO: $n=8$. e-g Assessment of spatial memory in the Barnes maze. e Schematics of the maze and averaged latencies to escape through the tunnel in each of the four sequential daily training sessions. Control: $n=30 ; 4 \mathrm{KO}: n=13 ; 5 \mathrm{KO}: n=10 ;$ DKO: $n=30$. $\mathbf{f}$ Total travel distances in the maze during training. $\mathbf{g}$ Memory retrieval in the probe test with escape tunnel removed. Graph shows averaged percentages of time spent in the correct (target) and other quadrants of the maze. Control: $n=30 ; 4 \mathrm{KO}: n=12 ; 5 \mathrm{KO}: n=$ 11; DKO: $n=29$. $\mathbf{h}$ Acquisition and extinction of associative fear memory. Mice were allowed to habituate $(H)$ and then subjected to CFC in Context $A$. Freezing was measured on a daily basis in the same or irrelevant context (Context B). Control: $n=15 ; 4 K O: n=14 ; 5 K O: n=11 ;$ DKO: $n=11$. i Conditional place preference (CPP) test. Graph shows averaged times spent on cocaine-paired floor. Control: $n=27 ; 4$ KO: $n=13 ; 5 K O: n=6 \varsigma ;$ DKO: $n=29$. All quantifications are represented as Mean \pm SEM. ${ }^{*} p<0.05$ (defined by $t$-test and ANOVA). Actual $p$-values are indicated in each graph (vertical for $t$-test and horizontal for ANOVA). § No sex-related differences in behavior were observed with exception of HDAC5-deficient mice in the CPP test, where only females had preference for cocaine-paired floor

escape latencies and travel in the Barnes maze and freezing in the irrelevant context (Fig. 3e, f, h; compare freezing in Context $\mathrm{A}$ and Context B).

As activity of HDAC5 in the nucleus accumbens is thought to affect conditional place preference ${ }^{35}$, we also studied this positive valence behavior in our mouse lines. We found that
$H D A C 5^{-/-}$mutants had no discrimination between regular and cocaine-paired floors, although this defect was only pronounced in males. By contrast, place preference of Hdac 4 flox/ flox/Emx $1^{\text {IRES-Cre }}$ males and females and $\mathrm{Hdac5}^{-/-}$females was similar to that of control animals, but it was completely abolished in DKOs of both genders (Fig. 3i). 


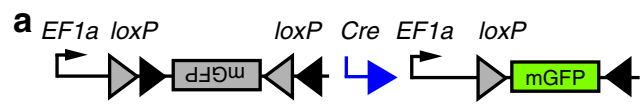

b

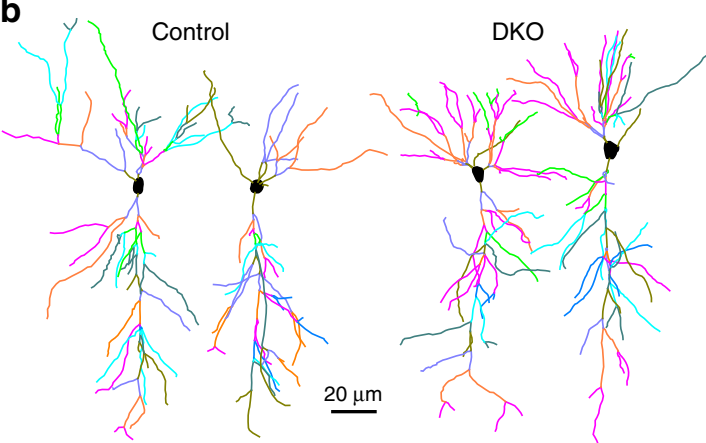

C

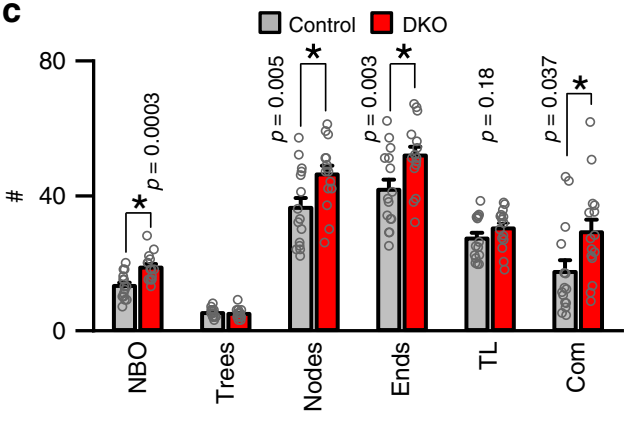

d

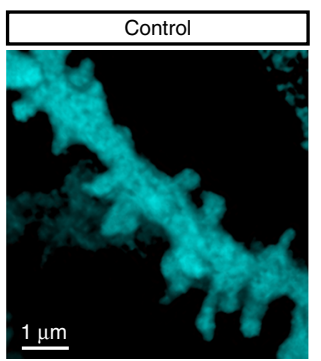

$\mathbf{f}$
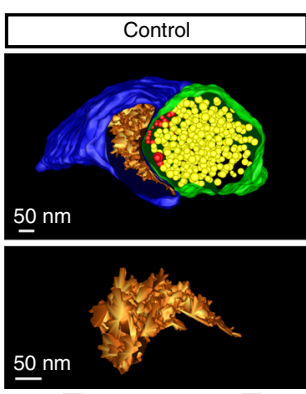

$\square$ Axon termina
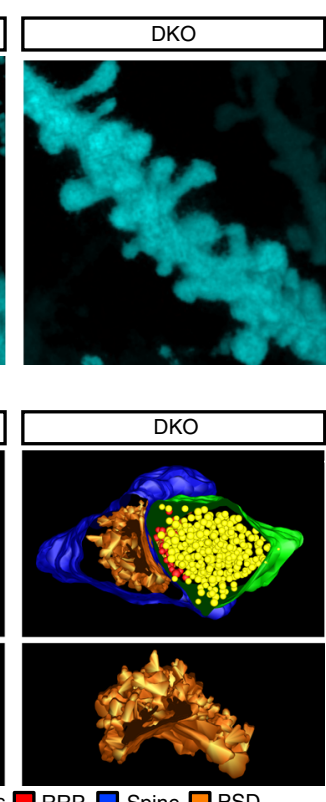

e
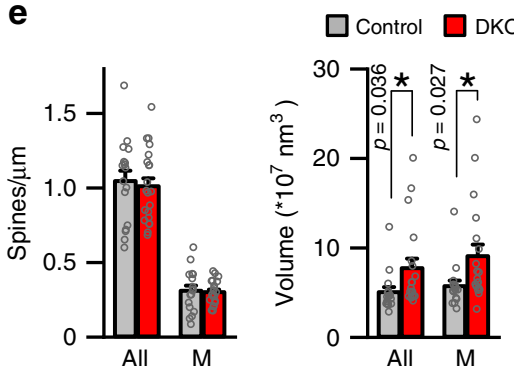

g

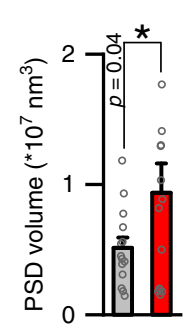

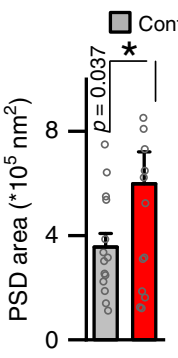

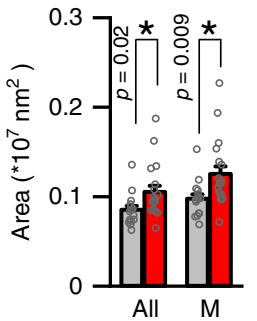

$\square \mathrm{DKO}$

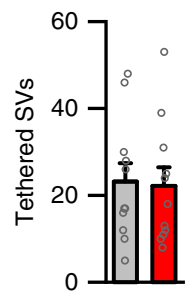

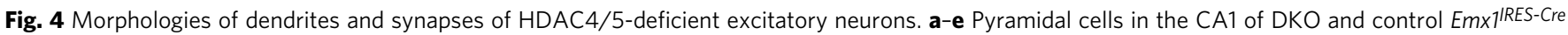
mice were visualized by Cre-inducible expression of membrane-bound GFP from an adeno-associated virus (AAV DJ DIO-mGFP). Animals were injected with AAVs at p45 and analyzed by confocal imaging of brain sections at p60. a Schematic diagram of AAV recombination. $\mathbf{b} 3 \mathrm{D}$ reconstructions of dendritic trees of individual glutamatergic neurons. c Quantifications of branch orders (NBO), trees, nodes, ends, tree length ( $T L$, $\mu$ m $\times 100$ ), and complexity indexes (Com, a.u. ×10,000). Averaged values are from 3 mice/15 neurons per genotype. d Pseudo-colored images of postsynaptic spines on proximal dendrites in CA1sr. e Linear densities, surface areas, and volumes of all and mushroom (M)-type spines. Control: $n=3$ mice/17 neurons; DKO: $n$ =3/21. f, $\mathbf{g}$ EM tomography analysis of architectures of glutamatergic synapses in CA1sr. $\mathbf{f}$ Top panels: 3D reconstructions of spines, postsynaptic densities (PSDs), nerve terminals, and synaptic vesicles (SVs). Bottom panels: isolated 3D reconstructions of PSDs. $\mathbf{g}$ Average volumes and surface areas of PSDs, total SV pools, and numbers of vesicles tethered to active zones (RRP). Control: $n=3$ mice/14 synapses; DKO: $n=3 / 14$. All quantifications are represented as Mean \pm SEM. ${ }^{\star} p<0.05$ (defined by $t$-test). Actual $p$-values are indicated in each graph

To begin to investigate how cognitive deficits of $\mathrm{DKO}$ mice correlate with changes in neural circuit structure and function, we analyzed the morphologies of glutamatergic neurons in the hippocampus. We sparsely labeled CA1 pyramidal cells in vivo via stereotactic injection of the Cre recombinase-inducible viral tracer, AAV DJ DIO-mGFP (Fig. 4a), collected confocal image stacks from fixed brain sections, and reconstructed single neurons for three-dimensional (3D) view. In agreement with our analysis of the anatomy of the hippocampus (Fig. 2e), genetically tagged HDAC4/5-deficient neurons were appropriately positioned in CA1sp and had fully polarized dendrites (Fig. 4b). Yet, quantitative tracing of dendritic trees revealed significantly higher numbers of branch orders, nodes, ends, and overall complexity of arborization (Fig. 4c). We then imaged dendrites in CA1sr at higher magnification to inspect their spines. Although the linear densities of all and mature mushroom-type spines were the same in DKOs and Emx1 ${ }^{I R E S-C r e}$ controls, DKO neurons had significantly larger spine sizes (Fig. 4d, e).

Considering that spines undergo transient enlargement during long-term synaptic potentiation (LTP), which also coincides with expansion of postsynaptic densities (PSDs) comprising neurotransmitter receptors, scaffolding molecules, and signaling enzymes $^{44,45}$, we analyzed the ultrastructural organization of glutamatergic synapses in the CA1sr by serial electron microscopy. To this end, tomographic images were acquired from $300 \mathrm{~nm}$ sections with $0.5^{\circ}$ tilt increments at $\times 22,500$ magnification and terminals with opposed spines that contain characteristic PSDs were reconstructed for $3 \mathrm{D}$ view from serial stacks. Synapses 
of HDAC4/5-defienct neurons were appropriately compartmentalized and had unaltered total pools of neurotransmitter vesicles as well as vesicles tethered to presynaptic active zones, but their PSDs were markedly bigger (Fig. 4f, g). Thus, class IIa HDACs restrict dendritic fields of hippocampal pyramidal neurons and affect their spine architectures in vivo.

As the next step, we relied on electrophysiological whole-cell recordings from acute hippocampal slices to evaluate intrinsic excitability of CA1 neurons and synaptic transmission in the circuit. Recordings in current-clamp mode showed that HDAC4/ 5 -deficient cells had no changes in resting membrane potentials and action potential firing in response to depolarization (Fig. 5a, b). However, measurements of miniature and evoked excitatory postsynaptic currents (EPSCs) demonstrated that loss of the two nuclear repressors leads to augmentation of glutamatergic synaptic strength. Neurons in slices from DKO mice had $\sim 2$-fold higher frequencies of spontaneous miniature AMPA-type EPSCs and elevated AMPA/NMDA ratios of evoked responses (Fig. 5c-f). Together with viral tracing of dendrites of individual neurons, these functional abnormalities indicate both higher numbers of excitatory inputs (due to larger receptive fields) and persistent postsynaptic potentiation, as augmented AMPA/ NMDA ratio is a signature of $\operatorname{LTP}^{46,47}$. Moreover, we did not detect significant shifts in profiles of synchronous EPSC facilitation/depression during high-frequency stimulus trains, which implies that presynaptic release probability and short-term plasticity of glutamatergic synapses formed by the CA3 afferents were normal (Fig. 5g, h).

Our results thus far provide several important insights into how class IIa HDAC signaling regulates neural circuits at cellular, subcellular, and molecular levels, but the contribution of these repressors and their downstream target genes to memory coding is still unclear. Indeed, given the nature of genetic manipulation used in DKO mice, their cognitive defects may be attributed to atypically broad induction of ERGs during learning, pre-existing abnormalities in neuronal wiring, synapse structures and synaptic transmission that reflect repetitive mis-regulation of experiencedependent transcriptional programs and preclude the appropriate processing of sensory information, or both. Likewise, the current understanding of physiological roles of ERGs in the mammalian brain is almost exclusively based on outcomes of prolonged lossor gain-of-function of these genes in animal models, as even drug-inducible Cre drivers and promoters offer relatively poor temporal control ${ }^{1,16-18}$. Although the necessity of gene expression for learning and memory is also demonstrated by pioneering experiments with pharmacological inhibitors of transcription and translation $^{2}$, these inhibitors lack cellular specificity and globally affect all genes. We therefore devised a strategy for acute transcriptional repression in brains of live and behaving mice that leverages direct stabilization of nuclear HDAC4 with a small molecule.

We fused the constitutively nuclear gain-of-function HDAC43SA mutant with the ecDHFR-based N-terminal destabilizing domain (DD), which mediates instant proteasomal degradation of newly synthesized polypeptides ${ }^{48}$. The decay of this DDnHDAC4 fusion protein can be blocked by the biologically "inert" antibiotic, Trimethoprim (TMP), which rapidly (within minutes) diffuses through tissues, penetrates the blood-brain barrier, and binds to the DD tag with high affinity ${ }^{48,49}$ (Fig. 6a). The construct also contained the C-terminal FLAG epitope for detection with antibodies (Supplementary Fig. 8a). To introduce DD-nHDAC4 into genetically defined neurons, we generated the "floxed" Rosa26 allele that permits expression under the control of the CAG promoter in a Cre recombinase-dependent manner (R26 floxStop-DD-nHDAC4 (Fig. 6a)). This allele was then crossed with a well-characterized forebrain-specific driver, Camk $2 \alpha^{C r e 50}$, to induce DD-nHDAC4 in fully differentiated glutamatergic neurons.

Immunoblotting and immunofluorescent imaging analyses of brains of adult R26floxStop-DD-nHDAC4/Camk2 $\alpha$ Cre mutants and their control Cre-negative littermates confirmed that DDnHDAC4 was only expressed in the forebrain in the presence of Cre, was effectively regulated by TMP, and was localized in neuronal nuclei. The protein was nearly undetectable without drug treatments, but its stability was restored within $2-3 \mathrm{~h}$ after single intraperitoneal injections of TMP and was maintained for several hours (Fig. 6b-e). Hence, we were able to achieve temporal control of nuclear class IIa HDAC activity in vivo that is at least an order of magnitude faster than temporal control of conventional and inducible promoter-based systems whose kinetics are dictated by rates of mRNA synthesis and degradation. Consistent with the previously described pattern of Camk $2 \alpha \mathrm{Cre}$ driven recombination, we observed robust expression of DDnHDAC4 in the hippocampus, the cerebral cortex, and the amygdala (Supplementary Fig. 8b).

To determine how unstable and TMP-bound DD-nHDAC4 forms affect experience-dependent transcription of class IIa HDAC target genes, we subjected R26loxStop-DD-nHDAC4/ Camk $2 \alpha^{C r e}$ mice to CFC and monitored the expression of Fos and Egrl in the hippocampus by immunofluorescent imaging $1.5 \mathrm{~h}$ later. Analysis of vehicle-treated animals showed that the system has negligible drug-independent background, whereas administration of TMP suppressed the ERG induction in trained mice (Fig. 6f-h and Supplementary Fig. 8c). Hence, this chemicalgenetic technique is suitable for transient cell-type-specific silencing of genes that become induced in response to sensory input and, unlike other genes, exhibit narrow peaks of transcriptional activity and encode mRNAs and proteins with short lifespans.

Having validated the new approach, we asked how acute repression of experience-dependent gene programs in glutamatergic neurons impacts associative fear memory using two different protocols in which animals were given TMP either $3 \mathrm{~h}$ before CFC or $3 \mathrm{~h}$ before contextual memory retrieval. In each case, freezing was measured $24 \mathrm{~h}$ after training (Fig. 6i). Both sets of experiments included vehicle- and TMP-treated $R 26$ floxstop-DD$n H D A C 4 / C a m k 2 \alpha$ Cre and Cre-negative R26loxStop-DD-nHDAC4 mice to account for potential undesired effects of the antibiotic itself and background activity of TMP-free DD-nHDAC4. Strikingly, transient stabilization of DD-nHDAC4 prior to learning strongly impaired memory, as evidenced by $\sim 3$-fold reduction of freezing times of TMP-injected R26loxStop-DD-nHDAC4/Camk $2 \alpha$ Cre mice (Fig. 6j). This deficit was not due to prolonged baseline leak of the system, as vehicle alone had no measurable effect. Moreover, TMP treatments did not affect freezing responses in the absence of Camk $2 \alpha^{C r e}$, excluding the possibility of off-target interactions of the drug (Fig. 6j). Finally, stabilized DD-nHDAC4 had no detectable effect on freezing of pre-conditioned animals that received TMP following CFC, suggesting their memory retrieval was intact (Fig. 6k). Hence, experience-dependent transcriptional programs are required for memory acquisition, but these programs may become dispensable when a stable memory is formed.

\section{Discussion}

In summary, this study elucidates the function of two homologous nuclear repressors, reveals a mechanism that limits experience-dependent expression of genes essential for plasticity of neural circuits, and provides insight into how these genes contribute to memory encoding.

Over the past few years, HDACs have attracted considerable interest as potential therapeutic targets, prompting the design and 
a
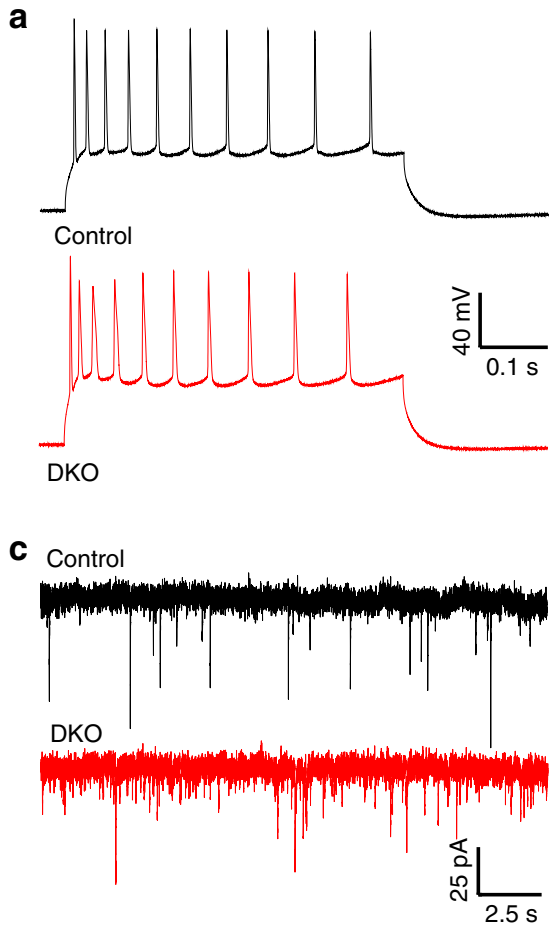

e

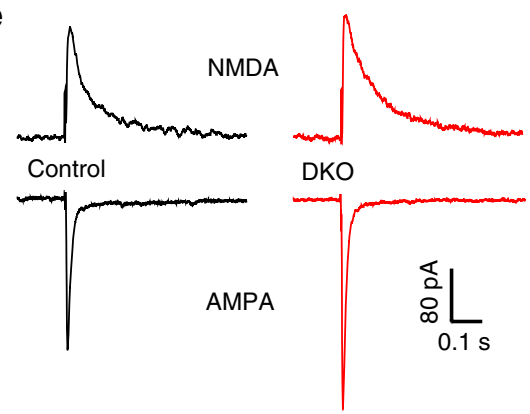

g Control

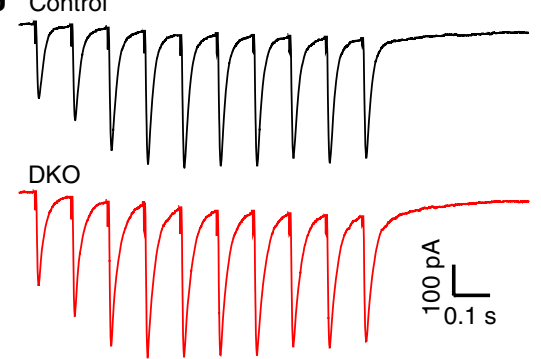

b

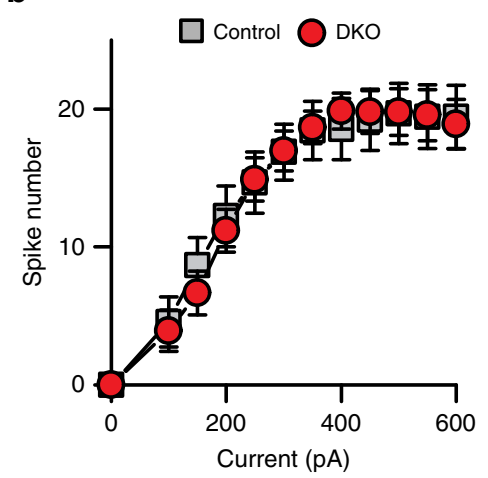

d

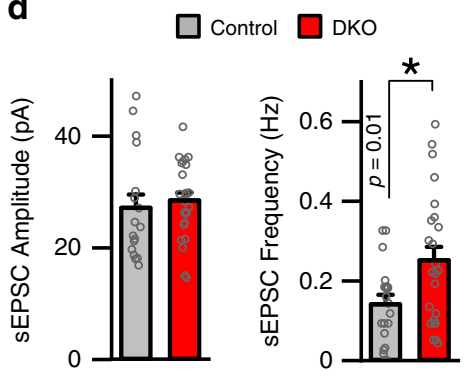

f

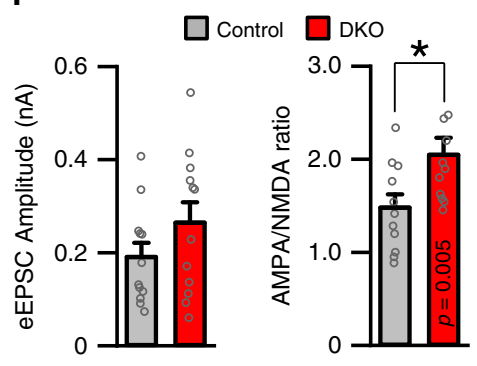

h

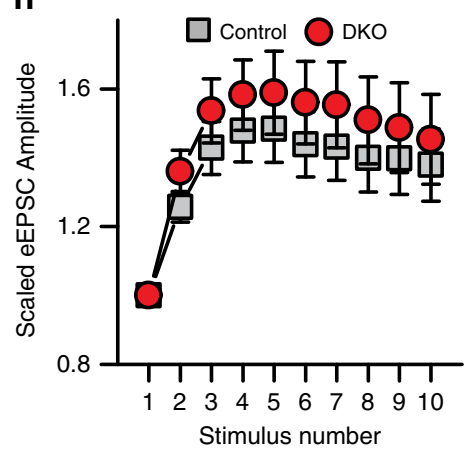

Fig. 5 Physiological properties of HDAC4/5-deficient excitatory neurons. Intrinsic excitability and synaptic strength of CA1 pyramidal cells were assessed by electrophysiological whole-cell recordings from acute brain slices from p20 mice. a Sample traces of depolarization-induced action potentials monitored in current-clamp mode. $\mathbf{b}$ Averaged numbers of action potentials, plotted as a function of stimulus intensity. Control: $n=5$ mice/18 neurons; DKO: $n=3 /$ 27. c Sample traces of spontaneous miniature excitatory postsynaptic currents (sEPSCs) recorded in voltage-clamp mode. Holding potentials were -70 $m V$. d Averaged sEPSC amplitudes and frequencies. Control: $n=5$ mice/20 neurons; DKO: $n=5 / 26$. e Traces of evoked AMPA (inward) and NMDA (outward) excitatory postsynaptic currents (eEPSCs) monitored at -70 and $+40 \mathrm{mV}$ holding potentials, respectively, in the presence of the GABA receptor blocker, Picrotoxin $(50 \mu \mathrm{M})$. Synaptic responses were triggered by electrical stimulation of CA3 afferents in the Schaffer collateral path. f Averaged AMPA eEPSC amplitudes (left) and AMPA/NMDA ratios (right). Control: $n=3$ mice/11 neurons; DKO: $n=3 / 11$. g Sample traces of AMPA eEPSC elicited by repetitive stimulation at $10 \mathrm{~Hz}$. h Averaged eEPSC amplitudes during $10 \mathrm{~Hz}$ trains, normalized to amplitudes of first responses. Control: $n$ $=5$ mice $/ 22$ neurons; DKO: $n=5 / 22$. All quantifications are represented as Mean \pm SEM. ${ }^{\star} p<0.05$ ( $t$-test) 
a

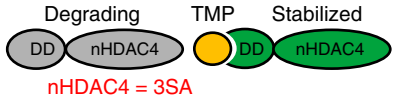

b

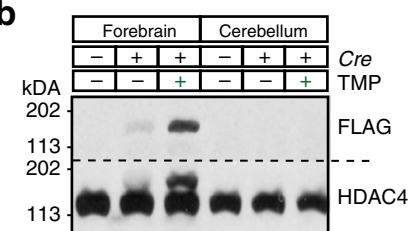

c
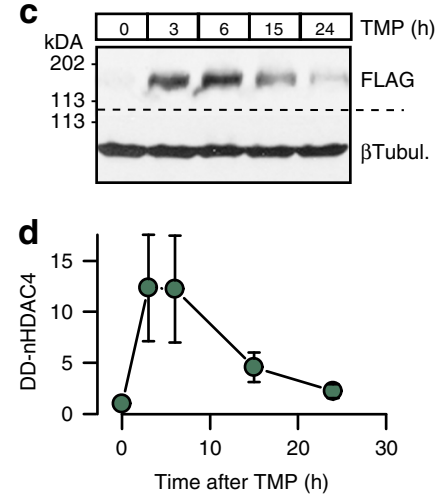

$R 26 \rightarrow \operatorname{loxP} \quad$ Cre $R 26 \rightarrow \operatorname{lox} P$
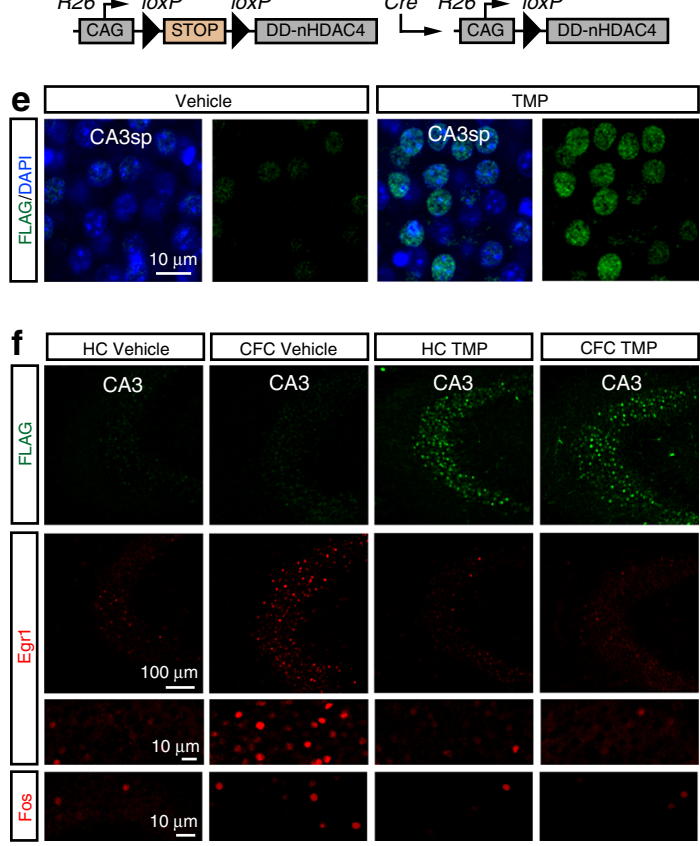

g
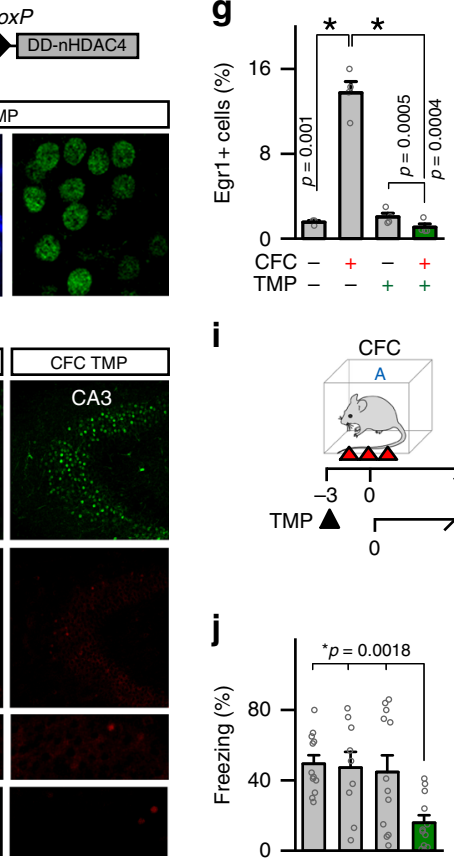

i

j h
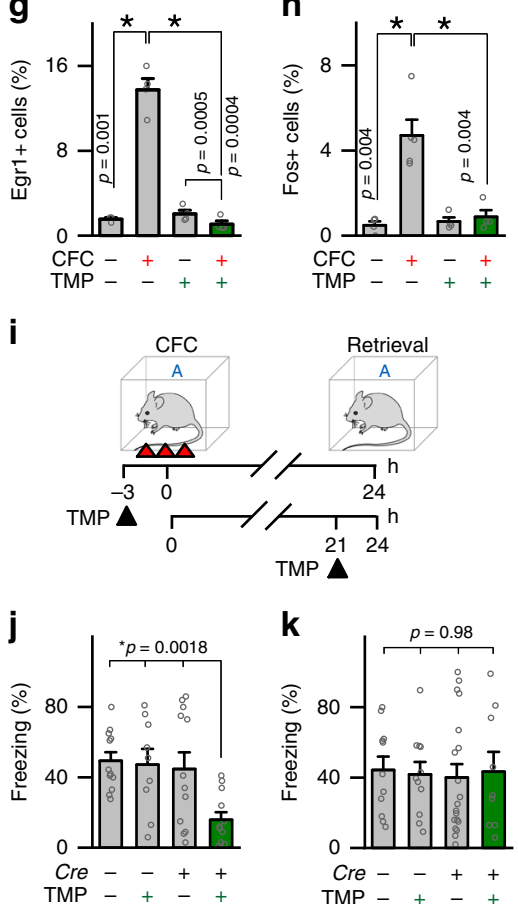

k

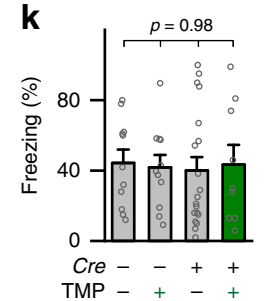

Fig. 6 Acute chemical-genetic control of nuclear repression with destabilized HDAC4. a Schematics of stabilization of DD-nHDAC4 protein with TMP and Rosa26 allele for Cre-dependent expression of DD-nHDAC4 form the CAG promoter (R26floxStop-DD-nHDAC4). See also Supplementary Fig. 8a. b-k DDnHDAC4 was introduced into excitatory forebrain neurons by crossing R26floxStop-DD-nHDAC4 mice with Camk $2 \alpha$ Cre. TMP-lactate (300 $\mu \mathrm{g} / \mathrm{gm}$ body weight) or control vehicle solutions were administered via intraperitoneal injections. b Brains were isolated at $\mathrm{p} 603 \mathrm{~h}$ after drug delivery. Cortical and cerebellar protein extracts were probed by immunoblotting with antibodies against FLAG or HDAC4. c, d Time course of DD-nHDAC4 stabilization in the cortex. Immunoblot of samples isolated at different intervals after TMP injection (c) and quantifications of DD-nHDAC4 levels (d, r.u.) are shown. $n=3-4$ mice/ time point. e Images of DD-nHDAC4 immunofluorescence in CA3sp of vehicle and TMP-treated R26floxStop-DD-nHDAC4/Camk2 $\alpha^{\text {Cre }}$ mice ( $3 \mathrm{~h} \mathrm{post} \mathrm{injection).}$ See also Supplementary Fig. 8b. f-h Stabilized DD-nHDAC4 represses ERGs. R26floxStop-DD-nHDAC4/Camk2 $\alpha^{\text {Cre }}$ mutants were given single doses of vehicle or TMP. Animals were maintained in home cages (HC) or subjected to CFC ( $3 \mathrm{~h}$ post-injection). After $1.5 \mathrm{~h}$, brains were sectioned and labeled with antibodies against FLAG, Egr1, or Fos. Panels show images of the CA3 (f) and averaged densities of Erg1/Fos-positive cells in this area (g, $\mathbf{h})$. See also Supplementary Fig. 8c. i-k Effects of TMP-inducible nuclear repression on associative memory. p60 R26floxStop-DD-nHDAC4/Camk2 $\alpha$ re and Cre-negative R26floxStop-DD-nHDAC4 mice were treated with vehicle or TMP prior to CFC or prior to contextual memory retrieval, as depicted in $\mathbf{i}$. $\mathbf{j}$ Freezing of animals that were given drugs $3 \mathrm{~h}$ before learning. Control + vehicle: $n=12$; Control + TMP: $n=9$; DD-nHDAC4 + vehicle: $n=12 ;$ DD-nHDAC4 + TMP: $n=12$. k Freezing of animals that were given drugs $3 \mathrm{~h}$ before memory recall. Control + vehicle: $n=11$; Control + TMP: $n=11 ;$ DD-nHDAC4 + vehicle: $n=19$; DDnHDAC4 + TMP: $n=9$. All quantifications are represented as Mean \pm SEM. ${ }^{*} p<0.05$ ( $t$-test and ANOVA). Actual $p$-values are indicated in each graph (vertical for $t$-test and horizontal for ANOVA)

clinical implementation of small molecule inhibitors ${ }^{21,51-53}$. However, the precise roles of class IIa HDACs in the nervous system have remained unclear, despite the evidence that loss or mis-localization of these proteins may lead to neurological abnormalities in model organisms and humans ${ }^{25-28,30,32,33,35}$. Unlike constitutively nuclear class I HDACs, vertebrate class IIa HDACs are unable to deacetylate histones due to evolutionarily acquired Tyrosine to Histidine substitution in the active site of the C-terminal enzymatic domain ${ }^{20,24}$. The hypothesis that neuronal HDAC4 functions as a transcriptional repressor has been supported by previous findings in several laboratories, including ours, demonstrating that this protein interacts with TFs CREB, MEF2, and SRF, and silences multiple genes when artificially forced to neuronal nuclei in vitro $25,29,32,33$. Yet, this hypothesis could not be reconciled with cytoplasmic localization of HDAC4 across the normal brain and perplexing lack of changes in transcription in brains of Hdac4 knockout mice $26,30,31,41$. HDAC5 has been recently shown to regulate drug seeking by repressing Npas4 in the mouse striatum independently of MEF2, although this conclusion was largely based on viral overexpression of nuclear constructs ${ }^{35}$. To date, the physiological relevance of HDAC5 and mechanism of its action in other circuits were also poorly understood.

In the first part of our work, we showed that HDAC4 is briefly imported to neuronal nuclei during associative learning, and that HDAC4 and HDAC5 control the same group of genes, including ERGs. Furthermore, we found that both repressors inhibit a synthetic activity-dependent promoter with binding sites for CREB, MEF2, and SRF, and their simultaneous ablation in excitatory forebrain neurons leads to anxiety and deficits in memory that could not be detected in single KOs. Lastly, we showed that DKO mutants exhibit a broader expression of ERGs after novel experience, as well as altered molecular profiles, morphologies, and synaptic strength of hippocampal neurons. Taken together, these results suggest that HDACs 4 and 5 redundantly act in the same pathway that restricts the induction of activity-dependent transcriptional programs, thereby limiting the numbers of neurons that undergo structural and/or functional plasticity in response to sensory input. In conjunction with reports that documented aberrant nuclear retention of HDAC4 in cellular and animal models of Parkinson's disease and Ataxia telangiectasia ${ }^{32,33}$, our results also imply that class IIa HDACs may exert their effects via 
different mechanisms under the physiological and pathological conditions depending on kinetics of nuclear import/export. In other words, HDACs 4 and 5 are intrinsically capable of repressing a broad spectrum of targets and they evidently do so upon irreversible nuclear entry (Fig. 1g) ${ }^{23,25,33}$, but their physiological effects in the brain appear to be strongly biased toward transiently expressed genes with narrow peaks of transcriptional activity and rapidly degrading mRNAs.

A second important aspect of our findings is that they emphasize the critical roles of both acute induction of transcription and spatiotemporal precision of this process for memory formation. Among class IIa HDAC effectors identified in our unbiased genome-wide RNA-seq screens, ERGs Arc, Nr4al, and Npas4 are known to be necessary for synaptic plasticity and memory ${ }^{16,54-61}$. Moreover, the essential roles of CREB, MEF2, and SRF in memory storage have been proven beyond any doubt ${ }^{2,14,62-64}$. Although the molecular events underlying behavioral phenotypes of repressor-deficient DKO mice are likely complex, it is reasonable to postulate that, at a circuit level, the cognitive deficits of these mice reflect the lack of cellular specificity of information coding that involves long-term changes in wiring and/or synaptic properties of sparsely distributed neural ensembles. On the other hand, our experiments with mice carrying a destabilized TMP-inducible nuclear HDAC4 imply that memory acquisition requires de novo transcription within a narrow window after sensory input. From a technical standpoint, these experiments validate a chemical-genetic approach that can be exploited for a broad range of studies in the future. For example, it is feasible to apply targeted expression of destabilized proteins for acute cell-type-specific genome editing, induction of TFs, or regulation of neuronal wiring and synaptic strength in live and behaving animals.

Although genetic tools leveraging Fos and other ERG promoters are becoming increasingly popular for tracing and optogenetic manipulation of neurons with a history of correlated activity elicited by sensory cues ${ }^{6-11,65}$, recent imaging studies have shown that, in the parietal cortex, activity patterns may also shift over time ${ }^{66}$. Dynamic spatiotemporal control of ERG programs by class IIa HDACs described herein may contribute to this network flexibility for processing of new information and therefore should be taken into account for better understanding of fundamental principles of neural coding. It is important to note that HDACs 4 and 5 also translocate to neuronal nuclei after global block of NMDA receptors or glutamate release in vitro and in vivo $22,23,25,29$, and the circuit-level mechanisms of experiencedependent nuclear import of these repressors need to be further elucidated. It would be of interest to eventually extend the present work with simultaneous in vivo imaging of fluorescently tagged class IIa HDACs and calcium indicators, GCaMPs, although this task will require development of new mouse models.

As class IIa HDACs and ERGs are expressed throughout the brain and in various neuron types, including GABAergic

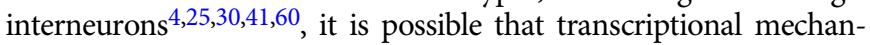
isms described here broadly regulate neural circuits and behavior. It is also noteworthy that vertebrate genomes have two other class II HDAC isoforms whose roles in the nervous system remain unclear: HDAC7, which appears to be predominantly expressed during early development, and HDAC9, which encodes a short protein that lacks the C-terminal deacetylase domain but also interacts with $\mathrm{MEF}^{20,25,67}$.

\section{Methods}

Mice were crossed to produce conditional lines, housed, and analyzed according to protocols approved by the Institutional Animal Care and Use Committee. Strains were maintained in mixed C56BL/6 and 129/Sv backgrounds. Studies were performed with animals of both genders. For each experiment, animal ages are indicated in the main text and/or figure legends. Ai9, Emx1 ${ }^{\text {IRES-Cre }}$, Camk $2 \alpha$ Cre, Hdac $4^{f l o x} f$ lox , and $H d a c 5^{-/-}$alleles were described previously ${ }^{37-40,50,68}$. To generate the R2floxStop-DD-nHDAC4 allele, the DD-nHDAC4 coding sequence (Supplementary Fig. 8a) was cloned into a targeting vector containing recombination arms, a ubiquitous CAG promoter, a loxP-flanked NEO cassette for positive selection, and a Diphtheria toxin cassette for negative selection. DD-nHDAC4 was placed downstream CAG and NEO, which served as a Cre recombinase-removable termination signal (Fig. 6a). The targeting vector was electroporated into embryonic stem (ES) cells and the positive clones were identified by PCR and Southern blotting. These clones were then used for blastocyst injection to produce chimeric mice. Chimeras were mated to WT mice and their offspring with confirmed germline transmission were used for subsequent crosses with Cre drivers.

Antibodies and expression vectors. Commercially available antibodies against the following proteins were used for immunofluorescent imaging and immunoblotting. HDAC4 (Santa Cruz, Cat\# sc-11418), HDAC4 (Sigma, Cat\# H9411), HDAC5 (Sigma, Cat\# H4538), $\beta$ Tubulin III (Sigma, Cat\# T2200), Fos (Santa Cruz, Cat\# sc-52G), Egr1 (Cell signaling, Cat\# 4153), NeuN (Millipore, Cat\# MAB377), Cux1 (Santa Cruz, Cat\# sc13024), Ctip2 (Abcam, Cat\# AB18465), Prox1 (Millipore, Cat\# AB5475), Calbindin (Swant, Cat\# CB38), and Cleaved Caspase3 (Cell Signaling, Cat\# 9661). LV and Adeno-associated virus (AAV) shuttle vectors encoding HDAC4-WT, HDAC4-3SA, E-SARE:EGFP, and DIO-mGFP were described previously 25,36,37,50. To generate LV vectors for expression of WT and 3SA of HDAC5, human coding sequences were amplified by PCR and subcloned downstream of the Synapsin promoter. HDAC5-3SA mutant contained Alanine substitutions of Serine residues S259, S279, and S498.

Neuronal cultures. Cortices of P1 pups were dissociated by trypsin digestion and seeded onto 24-well plates coated with poly-D-Lysine (Sigma). Cultures were maintained for 4 days in Neurobasal (Invitrogen) supplemented with fetal bovine serum, B-27 (Invitrogen), glucose, transferrin, and Ara-C (Sigma) followed by incubation in the serum-free medium.

Virus production and infection. Recombinant LVs were produced by cotransfection of human embryonic kidney 293T cells with corresponding shuttle vectors, and pVSVg and pCMV $\Delta 8.9$ plasmids that encode the elements essential for assembly and function of viral particles. Transfections were performed using the FuGENE reagent (Promega). Secreted viruses were collected $48 \mathrm{~h}$ later and cleared by brief centrifugation. Cultured neurons were infected at DIV2-5 with 100 $\mu \mathrm{l}$ of viral supernatants per $1 \mathrm{ml}$ of medium. This protocol was optimized to achieve $>95 \%$ infection efficiency. AAVs were produced in-house with shuttle vectors containing EF1 $a$ promoter, inverted terminal repeats, the WPRE element, and the hGH polyadenylation signal. To achieve cell-type-specific, Cre-inducible expression of fluorescent reporters, coding sequences were flanked by two pairs of loxP sites (DIO) and inserted downstream of a $1.26 \mathrm{~kb}$ EFla promoter in a $3^{\prime}-5^{\prime}$ orientation. These vectors were packed into AAV serotype DJ using published protocols ${ }^{50,69}$. Mice were stereotactically injected with $0.5 \mu \mathrm{l}$ of viral stocks via glass micropipettes $(10 \mu \mathrm{m}$ tip diameter) and returned to home cages until experiments.

Immunohistochemistry. Mice were anesthetized with isoflurane and perfused with $4 \%$ paraformaldehyde (PFA). The brains were incubated overnight in $0.5 \%$ PFA and sliced on vibratome in ice-cold phosphate-buffered saline (PBS). The $90 \mu \mathrm{m}$ thick, free-floating coronal sections (Bregma -1.4 to -2.5 ) were briefly boiled in $0.1 \mathrm{M}$ citrate for antigen retrieval. Sections were then washed three times in PBS, blocked for $1 \mathrm{~h}$ in a buffer containing 4\% bovine serum albumin, 3\% donkey serum, $0.1 \%$ Triton, and incubated overnight with primary antibodies diluted in blocking solution, followed by brief washes in PBS and $3 \mathrm{~h}$ incubation with corresponding fluorescently labeled secondary antibodies. Samples were washed again and mounted on glass slides.

Acquisition and analysis of confocal images. Specific brain regions were annotated using Allen Brain Atlas as the reference (http://mouse.brain-map.org/ static/atlas). Images were collected under the Nikon C2 confocal microscope with $\times 10, \times 20$, and $\times 40$ or $\times 60$ objectives. Thresholds and laser intensities were established for individual channels and equally applied to entire datasets. Conventional image analysis was conducted with Nikon Elements, FIJI, and Adobe Photoshop software packages. Digital manipulations were equally applied to all pixels. The $3 \mathrm{D}$ images of dendritic trees of single, virally labeled neurons were collected from 200 $\mu \mathrm{m}$ coronal sections at $0.2 \mu \mathrm{m} Z$-intervals. Neurons were subsequently reconstructed from serial stacks and analyzed in Neurolucida, as we have previously described $^{50}$. Spine densities were calculated alone individual fragments of proximal dendrites in CA1sr.

Electron microscopy. Mice were transcardially perfused with Ringer's solution followed by perfusion with $150 \mathrm{mM}$ cacodylate, $2.5 \%$ glutaraldehyde, $2 \%$ paraformaldehyde, and $2 \mathrm{mM} \mathrm{CaCl}_{2}$. The brains were fixed overnight in the same buffer at $4{ }^{\circ} \mathrm{C}$ and cut into $100 \mu \mathrm{m}$ coronal sections on vibratome. The slices were fixed overnight at $4^{\circ} \mathrm{C}$ and then washed for $1 \mathrm{~h}$ in $150 \mathrm{mM}$ cacodylate $/ 2 \mathrm{mM} \mathrm{CaCl}_{2}$ on 
ice. Sections ( $300 \mathrm{~nm}$ thick) were cut from the serial block-face scanning electron microscopy (SBEM)-stained specimens and collected on $50 \mathrm{~nm}$ Luxel slot grids (Luxel Corp., Friday Harbor, WA). The grids were coated with $10 \mathrm{~nm}$ colloidal gold (Ted Pella, Redding, CA) and imaged at $300 \mathrm{keV}$ on a Titan TEM (FEI, Hillsboro, OR). Double-tilt tilt-series were collected with $0.5^{\circ}$ tilt increments at $\times 22,500$ magnification on a $4 \mathrm{k} \times 4 \mathrm{k}$ Gatan Ultrascan camera. Tomograms were generated with an iterative scheme in the TxBR package ${ }^{70}$. Segmentation of synaptic structures and vesicle pools were performed in $\mathrm{IMOD}^{70,71}$.

qPCR and deep sequencing. mRNAs were extracted with the RNAeasy Kit (Qiagen). qPCR was performed with the following primers: Arc

Forward: 5'-GGTGAGCTGAAGCCACAAAT-3' Reverse: 5'-TTCACTGGTATGAATCACTGCTG-3' Fos

Forward: 5'- GGGACAGCCTTTCCTACTACC-3' Reverse: 5'- AGATCTGCGCAAAAGTCCTG-3' Egr1

Forward: 5' - CCTATGAGCACCTGACCACA-3' Reverse: 5'- AGCGGCCAGTATAGGTGATG-3' Nr4a1

Forward: 5'- TTGAGTTCGGCAAGCCTACC-3' Reverse: 5'- GTGTACCCGTCCATGAAGGTG-3' Npas4

Forward: $5^{\prime}$ - TTCACAGCTGAGGGGAAGTT-3'

Reverse: $5^{\prime}$ - TGTTGAATCGACAACGGAAA-3'

Gapdh

Forward: 5' - TCAACGGGAAGCCCATCA-3'

Reverse: 5' - CTCGTGGTTCACACCCATCA-3'

Conventional RNA-seq was performed at the TSRI Next Generation Sequencing Core on the Illumina HiSeq platform. The libraries were generated, barcoded, and sequenced according to the manufacturer's recommendations. Sequencing data were analyzed using a two-part in-house pipeline. First, gene expression levels across all samples were quantified with Salmon, a unique quasimapping approach for mapping sequences directly to a transcriptome ${ }^{72}$. Salmon also includes procedures to remove sample-specific technical biases that can arise during RNA-seq, including sequence-specific, position-specific, and fragment-GC content biases. Next, differential expression analysis was performed in R package DESeq $2^{73}$. DESeq2 first adjusts read counts based on a normalization factor that accounts for sample size. This is followed by dispersion estimates based on a negative binomial model, which accounts for genes with very few counts. Finally, a Wald's test was performed to calculate differential expression between the sample groups. Genes with an adjusted $p$-value of $<0.05$ and absolute fold change $>2$ were identified as significantly differentially expressed.

For single-cell RNA-seq, the brains were placed in oxygenated artificial cerebrospinal fluid (ACSF) containing $125 \mathrm{mM} \mathrm{NaCl}, 2.5 \mathrm{mM} \mathrm{KCl}, 2 \mathrm{mM}$

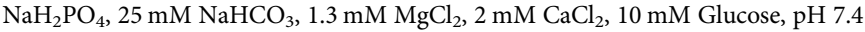
$(95 \% \mathrm{O} 2$ and $5 \% \mathrm{CO} 2)$. Sections $(300 \mu \mathrm{m})$ were cut on a vibratome (Leica VT1200S) and transferred into ice-cold Hibernate A/B27 medium $(50 \mathrm{ml}$ Hibernate A medium with $1 \mathrm{ml} \mathrm{B27}$ and $0.125 \mathrm{ml}$ Glutamax) containing $5 \mu \mathrm{g} / \mathrm{ml}$ actinomycin D (Sigma). Hippocampi were microdissected and incubated for 20 min on ice in Hibernate A/B27 medium containing $1 \mu \mathrm{M}$ TTX (Tocris), $100 \mu \mathrm{M}$ AP-V (Tocris), and $5 \mu \mathrm{g}$ actinomycin D (Sigma). Enzymatic digestion was performed in Hibernate A-Ca medium with $2 \mathrm{mg} / \mathrm{ml}$ papain, $1 \mu \mathrm{M}$ TTX, $100 \mu \mathrm{M}$ $\mathrm{AP}-\mathrm{V}, 25 \mu \mathrm{g}$ actinomycin $\mathrm{D}$ at $30^{\circ} \mathrm{C}$ for $40 \mathrm{~min}$. The tissues were then dissociated by gentle trituration through Pasteur pipettes with fire-polished tips. Single-cell suspensions were loaded on a four-layer OptiPrep gradient and centrifuged at $800 \times g$ for $15 \mathrm{~min}$ at $4^{\circ} \mathrm{C}$. Cell debris were removed by Debris Removal Solution (Miltenyi Biotec) following the manufacturer's instruction. Chromium scRNA-Seq reads were analyzed using Cell Ranger, a suite consisting of multiple pipelines for end to end analysis of single-cell data (https://support.10xgenomics.com). In brief, it uses a custom-built wrapper around Illumina's bcl2fastq (https://support. illumina.com) to demultiplex raw basecalls. This is followed by removal of duplicates using UMI counting. These preprocessed samples are aligned using $\mathrm{STAR}^{74}$, which performs splicing-aware alignment of reads to the genome. Aligned reads are then bucketed into exonic and intronic regions using a reference GTF file. Finally, read counts per gene are calculated and these values are used for downstream estimation of differentially expressed genes.

Electrophysiology. Mice were anesthetized with isoflurane. Brains were removed and placed into ice-cold oxygenated buffer $\left(95 \% \mathrm{O}_{2} / 5 \% \mathrm{CO}_{2}\right)$ containing $110 \mathrm{mM}$ Sucrose, $87 \mathrm{mM} \mathrm{NaCl}, 2.5 \mathrm{mM} \mathrm{KCl}, 0.5 \mathrm{mM} \mathrm{CaCl}_{2}, 7 \mathrm{mM} \mathrm{MgCl}, 25 \mathrm{mM}$ $\mathrm{NaHCO}_{3}, 1.25 \mathrm{mM} \mathrm{NaH}_{2} \mathrm{PO}_{4}$, and $20 \mathrm{mM}$ Glucose. Transverse, $350 \mu \mathrm{m}$-thick slices were cut with a vibratome and then allowed to recover for $\sim 1 \mathrm{~h}$ in oxygenated ACSF at $24^{\circ} \mathrm{C}$ prior to recording. Membrane potentials and synaptic currents were monitored in whole-cell mode using a Multiclamp 700B amplifier (Molecular Devices, Inc.). Recordings were performed at room temperature. For voltage-clamp experiments, the pipette solution contained $122.5 \mathrm{mM} \mathrm{C}_{6} \mathrm{H}_{12} \mathrm{O}_{7}, 122.5 \mathrm{mM} \mathrm{CsOH}$, $10 \mathrm{mM} \mathrm{CsCl}, 1.5 \mathrm{mM} \mathrm{MgCl} 2,5 \mathrm{mM} \mathrm{NaCl}, 1 \mathrm{mM}$ EGTA, $5 \mathrm{mM}$ HEPES, $3 \mathrm{mM}$ MgATP, $0.3 \mathrm{mM}$ NaGTP, $5 \mathrm{mM}$ QX-314, and $10 \mathrm{mM} \mathrm{Na}_{2}$ phosphocreatine $(\mathrm{pH}$
7.4). For current-clamp experiments, the pipette solution contained $120 \mathrm{mM} \mathrm{K}$ Gluconate, $20 \mathrm{mM} \mathrm{KCl}, 4 \mathrm{mM} \mathrm{Na} 2 \mathrm{ATP}, 0.3 \mathrm{mM} \mathrm{Na}_{2} \mathrm{GTP}, 4 \mathrm{mM} \mathrm{Na}_{2}$ phosphocreatine, $0.1 \mathrm{mM}$ EGTA, and $10 \mathrm{mM}$ HEPES (pH 7.4). Synaptic release was triggered by $1 \mathrm{~ms}$ current injections through the local extracellular stimulating electrode (FHC, Inc. CBAEC75). The frequency, duration, and magnitude of extracellular stimuli were controlled by Model 2100 Isolated Pulse Stimulator (A-M Systems, Inc.). Traces were analyzed offline with pClamp10 (Molecular Devices, Inc.) and Origin8 (Origin Lab) software packages.

Drug delivery. TMP-lactate (Sigma, Cat \#T0667) was reconstituted in PBS prior to each experiment $(30 \mathrm{mg} / \mathrm{ml})$ and administered to mice by intraperitoneal injections through a $29 \mathrm{~g}$ needle at a dose of $300 \mu \mathrm{g} / \mathrm{gm}$ body weight.

Behavior. Hindlimb clasping. Mice were grasped by tails and lifted for $10 \mathrm{~s}$. The scores were assigned using the following criteria, essentially as described ${ }^{75}: 0=$ hindlimbs are consistently splayed outward, away from the abdomen; $1=$ one hindlimb is retracted toward the abdomen for $>50 \%$ of the time suspended; $2=$ both hindlimbs are partially retracted toward the abdomen for $>50 \%$ of the time suspended; $3=$ hindlimbs are entirely retracted and touching the abdomen for $>50 \%$ of the time suspended.

Locomotor activity was measured for $2 \mathrm{~h}$ in polycarbonate cages $(42 \times 22 \times 20$ $\mathrm{cm})$ placed into frames $(25.5 \times 47 \mathrm{~cm})$ mounted with two levels of photocell beams at 2 and $7 \mathrm{~cm}$ above the bottom of the cage (San Diego Instruments, San Diego, $\mathrm{CA}$ ). These two sets of beams allow for the recording of both horizontal (locomotion) and vertical (rearing) behavior.

Vision was assessed in a stationary elevated platform surrounded by a drum with black and white striped walls. Mice were placed on the platform to habituate for $1 \mathrm{~min}$ and then the drum was rotated at 20 r.p.m. in one direction for $1 \mathrm{~min}$, stopped for $30 \mathrm{~s}$, and then rotated in the other direction for $1 \mathrm{~min}$. The total number of head tracks $\left(15^{\circ}\right.$ movements at speed of drum) was recorded. In our hands, mice that have intact vision track 5-25 times, whereas blind mice do not track at all.

Anxiety-like behavior was assessed using a light/dark transfer test, which capitalizes on the conflict between exploration of a novel environment and the avoidance of a brightly lit open field ${ }^{76}$. Mice were placed in the rectangular box divided by a partition into two environments. One compartment $(14.5 \times 27 \times 26.5$ $\mathrm{cm})$ was dark (8-16 lux) and the other compartment $(28.5 \times 27 \times 26.5 \mathrm{~cm})$ was highly illuminated ( $400-600$ lux) by a $60 \mathrm{~W}$ light source located above it. The compartments were connected by an opening $(7.5 \times 7.5 \mathrm{~cm})$ located at floor level in the center of the partition. The time spent in the light compartment and the number of transitions into the light compartment during 5 min sessions were measured as predictors of anxiety.

Spatial memory was examined in the Barnes maze ${ }^{42,77}$, a setup that consists of an opaque Plexiglass disc $75 \mathrm{~cm}$ in diameter elevated $58 \mathrm{~cm}$ above the floor by a tripod. Twenty holes, $5 \mathrm{~cm}$ in diameter, are located $5 \mathrm{~cm}$ from the perimeter and a black Plexiglass escape box $(19 \times 8 \times 7 \mathrm{~cm})$ is placed under one of the holes. Distinct spatial cues are located around the maze and are kept constant throughout the study. Acquisition: On the first day of testing, a training session was performed, which consisted of placing the animals in the escape box for $1 \mathrm{~min}$. At the beginning of each session, mice were then placed in the middle of the maze in a 10 $\mathrm{cm}$ high cylindrical black start chamber. After $10 \mathrm{~s}$ the start chamber was removed, a buzzer $(80 \mathrm{~dB})$ and a light (400 lux) were turned on, and mice were set free to explore the maze. The session ended when mice entered the escape tunnel or after 3 min elapsed. When mice entered the escape tunnel, the buzzer was turned off and animals were allowed to remain in the dark for $1 \mathrm{~min}$. If a mouse did not enter the tunnel by itself, it was gently put in the escape box for $1 \mathrm{~min}$. The tunnel was always located underneath the same hole (stable within the spatial environment), which was randomly determined for each mouse. Mice were tested once a day for 4 days for the acquisition portion of the study. Probe test: For the fifth test (probe test), the escape tunnel was removed and mice were allowed to freely explore the maze for $3 \mathrm{~min}$. The time spent in each quadrant was determined and the percent time spent in the target quadrant (the one originally containing the escape box) was compared with the average percent time in the other three quadrants. This is a direct test of spatial memory, as there is no potential for local cues to be used in the mouse's behavioral decision.

Associative memory was examined by using $\mathrm{CFC}^{3,9,10,78}$. Mice were allowed to explore the fear-conditioning boxes (Context A, Med Associates SD) for 3 min and were then subjected to four bursts of foot shocks $(0.55 \mathrm{~mA}, 1$ min inter-shock intervals). Memory tests consisted of one 3 min exposure to the training box or irrelevant box (Context B). Freezing was determined in $0.75 \mathrm{~s}$ bouts and expressed as percent time in the context.

Conditional place preference ${ }^{79}$ test involves pairing a distinct environmental context (floor type) with a motivationally significant event (cocaine injection). Rectangular Plexiglas black matte boxes $(42 \times 22 \times 30 \mathrm{~cm})$ divided by central partitions into two chambers of equal size $(22 \times 22 \times 30 \mathrm{~cm})$ were used. Distinctive tactile stimuli were provided in the two compartments of the apparatus. One chamber had no additional flooring and the other had lightly textured milkycolored flooring. During pre-conditioning and testing sessions, an aperture $(4 \times 4$ $\mathrm{cm})$ in the central partition allowed the animals to enter both sides of the apparatus. All testing occurred during the dark cycle under red light and was 
analyzed from video files using Noldus Ethovision software. The experiment consisted of three phases: pre-conditioning, conditioning, and testing in the following sequence. Day 1: Pre-conditioning phase with access to both compartments, Day 2-7: Conditioning phase-drug or saline administration followed by immediate confinement in one compartment of the place conditioning apparatus, and Day 8: Test with access to both compartments in the drug-free state. For the pre-conditioning phase, each animal was placed in one compartment of the apparatus and allowed to explore freely the entire apparatus for $30 \mathrm{~min}$. The time spent in each of the two compartments was measured. Mice showing unbiased exploration of the two sides of the apparatus (between $45 \%$ and $55 \%$ time spent on each side) were randomly assigned a chamber in which to receive cocaine. Mice showing biased exploration of either side were given cocaine in the least preferred compartment. Bias was observed in half of the mice tested and this was spread equally across each genotype and sex, and therefore did not confound the results. On the following 6 days, 30 min conditioning sessions were given in which animals were injected intraperitoneally with either saline or $10 \mathrm{mg} / \mathrm{kg}$ cocaine and immediately confined to one side of the apparatus (alternating these treatments and sides each day). In this way, each mouse experienced three pairings of cocaine with one of the apparatus sides. On the day after the final conditioning trial, each mouse was allowed to explore the entire apparatus in a non-drugged state for 30 min and the time spent in each of the two compartments of the conditioned place preference apparatus was recorded. The percent time spent in cocaine side was compared with the pre-conditioning session to examine the development of preference.

Quantifications and statistical analysis. Means and SEs were calculated in Origin8. $p$-values were determined with Student's $t$-test (for two groups) and analysis of variance (for multiple groups). Quantifications of gene expression levels, dendrite morphologies, spine densities and shapes, synapse ultra-structures, and all behavioral experiments were performed in a "blind" manner by investigators who were unaware of genotypes. Details, including sample sizes, can be found in figure legends.

Reporting summary. Further information on research design is available in the Nature Research Reporting Summary linked to this article.

\section{Data availability}

Source data are provided as a source data file. Additional relevant details can be obtained from the authors upon request.

Received: 15 March 2019 Accepted: 13 July 2019

Published online: 02 August 2019

\section{References}

1. West, A. E. \& Greenberg, M. E. Neuronal activity-regulated gene transcription in synapse development and cognitive function. Cold Spring Harb. Perspect. Biol. 3, a005744 (2011)

2. Kandel, E. R., Dudai, Y. \& Mayford, M. R. The molecular and systems biology of memory. Cell 157, 163-186 (2014)

3. Reijmers, L. G., Perkins, B. L., Matsuo, N. \& Mayford, M. Localization of a stable neural correlate of associative memory. Science 317, 1230-1233 (2007).

4. Hrvatin, S. et al. Single-cell analysis of experience-dependent transcriptomic states in the mouse visual cortex. Nat. Neurosci. 21, 120-129 (2018).

5. Tyssowski, K. M. et al. Different neuronal activity patterns induce different gene expression programs. Neuron 98, 530-546 (2018)

6. Cai, D. J. et al. A shared neural ensemble links distinct contextual memories encoded close in time. Nature 534, 115-118 (2016).

7. Ye, L. et al. Wiring and molecular features of prefrontal ensembles representing distinct experiences. Cell 165, 1776-1788 (2016).

8. Guenthner, C. J., Miyamichi, K., Yang, H. H., Heller, H. C. \& Luo, L. Permanent genetic access to transiently active neurons via TRAP: targeted recombination in active populations. Neuron 78, 773-784 (2013).

9. Liu, X. et al. Optogenetic stimulation of a hippocampal engram activates fear memory recall. Nature 484, 381-385 (2012).

10. Cowansage, K. K. et al. Direct reactivation of a coherent neocortical memory of context. Neuron 84, 432-441 (2014).

11. Tonegawa, S., Morrissey, M. D. \& Kitamura, T. The role of engram cells in the systems consolidation of memory. Nat. Rev. Neurosci. 19, 485-498 (2018).

12. Strange, B. A., Witter, M. P., Lein, E. S. \& Moser, E. I. Functional organization of the hippocampal longitudinal axis. Nat. Rev. Neurosci. 15, 655-669 (2014).

13. Chahrour, M. et al. MeCP2, a key contributor to neurological disease, activates and represses transcription. Science 320, 1224-1229 (2008).
14. Casadio, A. et al. A transient, neuron-wide form of CREB-mediated long-term facilitation can be stabilized at specific synapses by local protein synthesis. Cell 99, 221-237 (1999)

15. Kandel, E. R. The molecular biology of memory: cAMP, PKA, CRE, CREB-1, CREB-2, and CPEB. Mol. Brain 5, 14 (2012).

16. Ramamoorthi, K. et al. Npas4 regulates a transcriptional program in CA3 required for contextual memory formation. Science 334, 1669-1675 (2011).

17. Bridi, M. S. \& Abel, T. The NR4A orphan nuclear receptors mediate transcription-dependent hippocampal synaptic plasticity. Neurobiol. Learn. Mem. 105, 151-158 (2013).

18. Park, S. et al. Elongation factor 2 and fragile $\mathrm{X}$ mental retardation protein control the dynamic translation of Arc/Arg3.1 essential for mGluR-LTD Neuron 59, 70-83 (2008).

19. Josselyn, S. A., Kohler, S. \& Frankland, P. W. Finding the engram. Nat. Rev Neurosci. 16, 521-534 (2015).

20. Haberland, M., Montgomery, R. L. \& Olson, E. N. The many roles of histone deacetylases in development and physiology: implications for disease and therapy. Nat. Rev. Genet. 10, 32-42 (2009).

21. Parra, M. ClassI. Ia HDACs - new insights into their functions in physiology and pathology. FEBS J. 282, 1736-1744 (2015).

22. Chawla, S., Vanhoutte, P., Arnold, F. J., Huang, C. L. \& Bading, H. Neuronal activity-dependent nucleocytoplasmic shuttling of HDAC4 and HDAC5. J. Neurochem. 85, 151-159 (2003).

23. Schlumm, F., Mauceri, D., Freitag, H. E. \& Bading, H. Nuclear calcium signaling regulates nuclear export of a subset of class IIa histone deacetylases following synaptic activity. J. Biol. Chem. 288, 8074-8084 (2013).

24. Lahm, A. et al. Unraveling the hidden catalytic activity of vertebrate class IIa histone deacetylases. Proc. Natl Acad. Sci. USA 104, 17335-17340 (2007).

25. Sando, R. 3rd et al. HDAC4 governs a transcriptional program essential for synaptic plasticity and memory. Cell 151, 821-834 (2012).

26. Kim, M. S. et al. An essential role for histone deacetylase 4 in synaptic plasticity and memory formation. J. Neurosci. 32, 10879-10886 (2012)

27. Williams, S. R. et al. Haploinsufficiency of HDAC4 causes brachydactyly mental retardation syndrome, with brachydactyly type E, developmental delays, and behavioral problems. Am. J. Hum. Genet. 87, 219-228 (2010).

28. Fitzsimons, H. L., Schwartz, S., Given, F. M. \& Scott, M. J. The histone deacetylase HDAC4 regulates long-term memory in Drosophila. PLoS ONE 8, e83903 (2013)

29. Chen, Y., Wang, Y., Modrusan, Z., Sheng, M. \& Kaminker, J. S. Regulation of neuronal gene expression and survival by basal NMDA receptor activity: a role for histone deacetylase 4. J. Neurosci. 34, 15327-15339 (2014).

30. Chen, B. \& Cepko, C. L. HDAC4 regulates neuronal survival in normal and diseased retinas. Science 323, 256-259 (2009).

31. Mielcarek, M. et al. HDAC4 does not act as a protein deacetylase in the postnatal murine brain in vivo. PLoS ONE 8, e80849 (2013).

32. Li, J. et al. Nuclear accumulation of HDAC4 in ATM deficiency promotes neurodegeneration in ataxia telangiectasia. Nat. Med. 18, 783-790 (2012).

33. Lang, C. et al. Single-cell sequencing of iPSC-dopamine neurons reconstructs disease progression and identifies HDAC4 as a regulator of Parkinson cell phenotypes. Cell Stem Cell 24, 93-106 (2019).

34. Basu, J. \& Siegelbaum, S. A. The corticohippocampal circuit, synaptic plasticity, and memory. Cold Spring Harb. Perspect. Biol. 7, a021733 (2015)

35. Taniguchi, M. et al. HDAC5 and its target gene, Npas4, function in the nucleus accumbens to regulate cocaine-conditioned behaviors. Neuron 96 130-144 e136 (2017).

36. Kawashima, T. et al. Functional labeling of neurons and their projections using the synthetic activity-dependent promoter E-SARE. Nat. Methods 10, 889-895 (2013).

37. Sando, R. et al. Assembly of excitatory synapses in the absence of glutamatergic neurotransmission. Neuron 94, 312-321 e313 (2017).

38. Gorski, J. A., Balogh, S. A., Wehner, J. M. \& Jones, K. R. Learning deficits in forebrain-restricted brain-derived neurotrophic factor mutant mice. Neuroscience 121, 341-354 (2003).

39. Chang, S. et al. Histone deacetylases 5 and 9 govern responsiveness of the heart to a subset of stress signals and play redundant roles in heart development. Mol. Cell Biol. 24, 8467-8476 (2004).

40. Potthoff, M. J. et al. Histone deacetylase degradation and MEF2 activation promote the formation of slow-twitch myofibers. J. Clin. Invest. 117, 2459-2467 (2007)

41. Darcy, M. J., Calvin, K., Cavnar, K. \& Ouimet, C. C. Regional and subcellular distribution of HDAC4 in mouse brain. J. Comp. Neurol. 518, 722-740 (2010).

42. Bach, M. E., Hawkins, R. D., Osman, M., Kandel, E. R. \& Mayford, M. Impairment of spatial but not contextual memory in CaMKII mutant mice with a selective loss of hippocampal LTP in the range of the theta frequency. Cell 81, 905-915 (1995).

43. Sevenster, D., Visser, R. M. \& D’Hooge, R. A translational perspective on neural circuits of fear extinction: current promises and challenges. Neurobiol. Learn Mem. 155, 113-126 (2018). 
44. Bourne, J. N. \& Harris, K. M. Nanoscale analysis of structural synaptic plasticity. Curr. Opin. Neurobiol. 22, 372-382 (2012).

45. Bromer, C. et al. Long-term potentiation expands information content of hippocampal dentate gyrus synapses. Proc. Natl Acad. Sci. USA 115, E2410-E2418 (2018).

46. Makino, H. \& Malinow, R. AMPA receptor incorporation into synapses during LTP: the role of lateral movement and exocytosis. Neuron 64, 381-390 (2009).

47. Huganir, R. L. \& Nicoll, R. A. AMPARs and synaptic plasticity: the last 25 years. Neuron 80, 704-717 (2013).

48. Iwamoto, M., Bjorklund, T., Lundberg, C., Kirik, D. \& Wandless, T. J. A general chemical method to regulate protein stability in the mammalian central nervous system. Chem. Biol. 17, 981-988 (2010).

49. Sando, R. 3rd et al. Inducible control of gene expression with destabilized Cre. Nat. Methods 10, 1085-1088 (2013).

50. Pieraut, S. et al. Experience-dependent remodeling of basket cell networks in the dentate gyrus. Neuron 84, 107-122 (2014).

51. Abel, T. \& Zukin, R. S. Epigenetic targets of HDAC inhibition in neurodegenerative and psychiatric disorders. Curr. Opin. Pharm. 8, 57-64 (2008).

52. Bantscheff, M. et al. Chemoproteomics profiling of HDAC inhibitors reveals selective targeting of HDAC complexes. Nat. Biotechnol. 29, 255-265 (2011).

53. Graff, J. \& Tsai, L. H. The potential of HDAC inhibitors as cognitive enhancers. Annu Rev. Pharm. Toxicol. 53, 311-330 (2013).

54. Shepherd, J. D. et al. Arc/Arg3.1 mediates homeostatic synaptic scaling of AMPA receptors. Neuron 52, 475-484 (2006).

55. Alberi, L. et al. Activity-induced Notch signaling in neurons requires Arc/ Arg3.1 and is essential for synaptic plasticity in hippocampal networks. Neuron 69, 437-444 (2011).

56. McNulty, S. E. et al. Differential roles for Nr4a1 and Nr4a2 in object location vs. object recognition long-term memory. Learn. Mem. 19, 588-592 (2012).

57. Chen, Y. et al. Activity-induced Nr4al regulates spine density and distribution pattern of excitatory synapses in pyramidal neurons. Neuron 83, 431-443 (2014).

58. Jeanneteau, F. et al. The stress-induced transcription factor NR4A1 adjusts mitochondrial function and synapse number in prefrontal cortex. J. Neurosci. 38, 1335-1350 (2018).

59. Bloodgood, B. L., Sharma, N., Browne, H. A., Trepman, A. Z. \& Greenberg, M. E. The activity-dependent transcription factor NPAS4 regulates domainspecific inhibition. Nature 503, 121-125 (2013).

60. Spiegel, I. et al. Npas4 regulates excitatory-inhibitory balance within neural circuits through cell-type-specific gene programs. Cell 157, 1216-1229 (2014).

61. Sun, X. \& Lin, Y. Npas4: linking neuronal activity to memory. Trends Neurosci. 39, 264-275 (2016).

62. Martin, K. C. et al. Synapse-specific, long-term facilitation of aplysia sensory to motor synapses: a function for local protein synthesis in memory storage. Cell 91, 927-938 (1997).

63. Barbosa, A. C. et al. MEF2C, a transcription factor that facilitates learning and memory by negative regulation of synapse numbers and function. Proc. Natl Acad. Sci. USA 105, 9391-9396 (2008).

64. Cole, C. J. et al. MEF2 negatively regulates learning-induced structural plasticity and memory formation. Nat. Neurosci. 15, 1255-1264 (2012).

65. Okuyama, T., Kitamura, T., Roy, D. S., Itohara, S. \& Tonegawa, S. Ventral CA1 neurons store social memory. Science 353, 1536-1541 (2016).

66. Driscoll, L. N., Pettit, N. L., Minderer, M., Chettih, S. N. \& Harvey, C. D. Dynamic reorganization of neuronal activity patterns in parietal cortex. Cell 170, 986-999 e916 (2017).

67. Alchini, R. et al. Nucleocytoplasmic shuttling of histone deacetylase 9 controls activity-dependent thalamocortical axon branching. Sci. Rep. 7, 6024 (2017).

68. Madisen, L. et al. A robust and high-throughput Cre reporting and characterization system for the whole mouse brain. Nat. Neurosci. 13, 133-140 (2010).

69. McClure, C., Cole, K. L., Wulff, P., Klugmann, M. \& Murray, A. J. Production and titering of recombinant adeno-associated viral vectors. J. Vis. Exp. https:// doi.org/10.3791/3348 (2011).

70. Chen, R. et al. EPiK-a workflow for electron tomography in Kepler. Procedia Comput. Sci. 20, 2295-2305 (2014).

71. Kremer, J. R., Mastronarde, D. N. \& McIntosh, J. R. Computer visualization of three-dimensional image data using IMOD. J. Struct. Biol. 116, 71-76 (1996).

72. Patro, R., Duggal, G., Love, M. I., Irizarry, R. A. \& Kingsford, C. Salmon provides fast and bias-aware quantification of transcript expression. Nat. Methods 14, 417-419 (2017).
73. Love, M. I., Huber, W. \& Anders, S. Moderated estimation of fold change and dispersion for RNA-seq data with DESeq2. Genome Biol. 15, 550 (2014).

74. Dobin, A. et al. STAR: ultrafast universal RNA-seq aligner. Bioinformatics 29, 15-21 (2013)

75. Guyenet, S. J. et al. A simple composite phenotype scoring system for evaluating mouse models of cerebellar ataxia. J. Vis. Exp. https://doi.org/ 10.3791/1787 (2010).

76. Crawley, J. N. Behavioral phenotyping of transgenic and knockout mice: experimental design and evaluation of general health, sensory functions, motor abilities, and specific behavioral tests. Brain Res. 835, 18-26 (1999).

77. Barnes, C. A. Memory deficits associated with senescence: a neurophysiological and behavioral study in the rat. J. Comp. Physiol. Psychol. 93, 74-104 (1979).

78. Ramirez, S. et al. Creating a false memory in the hippocampus. Science 341, 387-391 (2013).

79. Cunningham, C. L., Patel, P. \& Milner, L. Spatial location is critical for conditioning place preference with visual but not tactile stimuli. Behav. Neurosci. 120, 1115-1132 (2006).

\section{Acknowledgements}

We thank Drs M. Mayford, L. Stowers, and G. Lippi for discussions and critical comments; Drs E. Olson, R. Shaw, and H. Bito for sharing mice and expression vectors; Dr K. Spencer for assistance with microscopy; Dr A. Roberts for assistance with behavioral experiments; and The Scripps Research Institute (TSRI) Next Generation Sequencing Core for deep sequencing and bioinformatics support. This study was funded by the NIH grants R01NS087026 (to A.M.), R01GM117049 (to A.M.), R01MH118442 (to A.M.) and P41GM301412 (to M.E.).

\section{Author contributions}

A.M. and Y.Z. conceived the study. Y.Z. generated mouse lines and personally carried out or supervised all molecular profiling, imaging, and behavioral experiments. M. H. performed electrophysiological recordings from acute brain slices. M. U. and K.T. assisted Y. Z. with confocal microscopy, quantitative real-time PCR, and inhouse tests of associative memory. Other behavioral experiments were performed at the TSRI core facility. E. Bus. and S.P. prepared samples for EM and collected tomograms. M.E. supervised EM studies. E. Beu. and D.B. traced genetically-tagged neurons in Neurolucida and analyzed 3D EM reconstructions in IMOD. A.M. wrote the manuscript.

\section{Additional information}

Supplementary Information accompanies this paper at https://doi.org/10.1038/s41467019-11409-0.

Competing interests: The authors declare no competing interests.

Reprints and permission information is available online at http://npg.nature.com/ reprintsandpermissions/

Peer review information: Nature Communications thanks the anonymous reviewers for their contribution to the peer review of this work. Peer reviewer reports are available.

Publisher's note: Springer Nature remains neutral with regard to jurisdictional claims in published maps and institutional affiliations.

Open Access This article is licensed under a Creative Commons Attribution 4.0 International License, which permits use, sharing, adaptation, distribution and reproduction in any medium or format, as long as you give appropriate credit to the original author(s) and the source, provide a link to the Creative Commons license, and indicate if changes were made. The images or other third party material in this article are included in the article's Creative Commons license, unless indicated otherwise in a credit line to the material. If material is not included in the article's Creative Commons license and your intended use is not permitted by statutory regulation or exceeds the permitted use, you will need to obtain permission directly from the copyright holder. To view a copy of this license, visit http://creativecommons.org/ licenses/by/4.0\%.

(C) The Author(s) 2019 